Pacific Journal of Mathematics

POINT SPECTRUM OF ERGODIC ABELIAN GROUP ACTIONS
AND THE CORRESPONDING GROUP-MEASURE FACTORS 


\title{
POINT SPECTRUM OF ERGODIC ABELIAN GROUP ACTIONS AND THE CORRESPONDING GROUP-MEASURE FACTORS
}

\author{
JUDith A. PACKeR
}

In this paper a study of certain conjugacy invariants of ergodic actions of countable discrete groups which appear in the analysis of the associated group measure factors is begun. In particular a certain maximal abelian subalgebra $S$ of the factor associated to the free ergodic measure-preserving action of a countable abelian group on a compact Lebesgue space is studied. The structure of the normalizer of this subalgebra is completely determined, and is shown to depend entirely on the point spectrum of the group action. We examine cocycles of the group action with values in a compact abelian group, the corresponding skew product actions and the related von Neumann algebras. Conditions that such an extension be ergodic, and the point spectrum distinct from the original action are obtained by examining the corresponding factors. For actions with pure point spectrum we examine cocycles with values in $S^{1}$ and the corresponding *-automorphism of the factor and determine necessary and sufficient conditions for the related Cartan subalgebra $S$ to be inner conjugate to its image under the cocycle automorphism. Applying our results to a particular group action with pure point spectrum, we are able to exhibit (modulo choice of an orbit equivalence) an uncountable family of Cartan subalgebras in the hyperfinite $\mathrm{II}_{1}$ factor, no two of which are inner conjugate.

Introduction. One of the original methods of constructing non-type I factors is the so-called "group-measure" construction due to Murray and von Neumann. This construction associates to any free ergodic action of a countable discrete group $G$ on a Lebesgue measure space $(X, \mu)$ a factor $F(X, G)$ acting on the Hilbert space $L^{2}(X \times G)$. To a large extent, the study of the relationship between the factor $F(X, G)$ and the ergodic action $(X, \mu, G)$ has emphasized the concept of orbit equivalence. Clearly a sufficient condition that free ergodic actions $\left(X_{1}, \mu_{1}, G_{1}\right)$ and $\left(X_{2}, \mu_{2}, G_{2}\right)$ give rise to *-isomorphic factors $F\left(X_{1}, G_{1}\right)$ and $F\left(X_{2}, G_{2}\right)$ is that the actions be orbit equivalent [4] (it has recently been shown in [2] that the reverse implication is not true in general). For example, if $G_{1}$ and $G_{2}$ are countable abelian groups acting freely and ergodically on the compact Lebesgue spaces $\left(X_{1}, \mu_{1}\right)$ and $\left(X_{2}, \mu_{2}\right)$ so as to preserve the finite measures $\mu_{1}$ and $\mu_{2}, F\left(X_{1}, G_{1}\right)$ and $F\left(X_{2}, G_{2}\right)$ will be $*$-isomorphic; both will be hyperfinite $\mathrm{II}_{1}$ factors. 
In this paper we attempt to move beyond the notion of orbit equivalence to initiate a study of how certain stronger invariants of the free ergodic action $(X, \mu, G)$ exhibit themselves in the inner structure of the factor $F(X, G)$. We shall mainly be restricting ourselves to the case where $G$ is abelian and preserves the finite measure $\mu$. The factor $F(X, G)$ contains as a subalgebra a copy of the left regular representation algebra of $G$. We denote this subalgebra by $S(X, G)$. Since the abelian group $G$ acts ergodically on $(X, \mu), S(X, G)$ is maximal abelian in $F(X, G)$. One naturally desires to know which unitary elements in $F(X, G)$ normalize $S(X, G)$; i.e., for which $U \in \mathscr{U}(F(X, G))$ does $U S(X, G) U^{*}=S(X, G)$.

This question is a special case of the following problem: if $(Y, \nu, G)$ is a quotient action of $(X, \mu, G)$, i.e., if there exists a $G$-equivariant surjective Borel map $\varphi: X \rightarrow Y$ with $\varphi * \mu=\nu$, then $F(Y, G)$ injects naturally as a subalgebra of $F(X, G)$. Which unitary elements in $F(X, G)$ normalize $F(X, G)$ ? We intend to investigate this more general problem in a subsequent paper. If $Y=$ pt., with $G$ acting on it trivially, then $F(X, G)=$ $S(X, G)$. For this situation we have the following result:

TheOREM 2.3. Let $G$ be a countable abelian group acting freely and ergodically on the compact Lebesgue space $(X, \mu)$ so as preserve the finite measure $\mu$. Then the normalizer of $S(X, G)$ in $F(X, G)$ which we define to be the subalgebra of $F(X, G)$ generated by the unitary elements which normalize $S(X, G)$, is equal to $F\left(Y_{d}, G\right)$, where $\left(Y_{d}, \nu, G\right)$ is the maximal quotient action of $(X, \mu, G)$ having pure point spectrum.

This result is closely related to a result of $\mathrm{P}$. Hahn [8], and was motivated by work of Nielsen [16] who considered the case where $(X, \mu, G)$ has continuous spectrum. We will elaborate on our debt to [8], [16] and [22] in the course of the proof.

In particular, we note that under the conditions of the theorem, $S(X, G)$ will be regular in $F(X, G)$, in the sense that the normalizing unitaries generate $F(X, G)$, if and only if $(X, \mu, G)$ has pure point spectrum. Since any von Neumann subalgebra of a $\mathrm{II}_{1}$ factor is the image of a faithful, normal conditional expectation, we see that under the conditions of the theorem $S(X, G)$ is a Cartain subalgebra (in the sense of [6]) if and only if $(X, \mu, G)$ has pure point spectrum.

We can use this fact to exhibit an uncountable family of Cartan subalgebras in the hyperfinite $\mathrm{II}_{1}$ factor, no two of which are inner conjugate. Our construction relies on the method of $*$-automorphisms of $F(X, G)$ associated to one-cocycles of $(X, \mu, G)$ with values in $S^{1}$ : a Borel 
map $\alpha: X \times G \rightarrow S^{1}$ which satisfies the cocycle identity:

$$
\alpha\left(x, g_{1}\right) \alpha\left(x g_{1}, g_{2}\right)=\alpha\left(x, g_{1} g_{2}\right) \quad \mu \text { a.e., } \quad \forall g_{1}, g_{2} \in G
$$

will give rise to a $*$-automorphism of $F(X, G)$, which we denote by $A_{\alpha}$. Then we have the following:

THEOREM 3.8. Let the countable abelian group $G$ act on the compact Lebesgue space $(X, \mu)$ so as to preserve the finite measure $\mu$, and suppose that $(X, \mu, G)$ has pure point spectrum. Let $\alpha$ be a one-cocycle $\alpha: X \times G \rightarrow$ $S^{1}$. Then $S(X, G)$ is inner conjugate to $A_{\alpha}(S(X, G))$ if and only if there exists a Borel function $f: X \rightarrow S^{1}$ and a character $\chi \in \hat{G}$ satisfying

$$
\alpha(x, g)=f(x g) \chi(g) f(x)^{-1} \quad \mu \text {. a.e. } \forall g \in G .
$$

The proof involves studying the point spectrum of certain skew-product extension of $(X, \mu, G)$; we thus have related results in these areas.

The uncountable family of pairwise non-inner conjugate Cartan subalgebras of the hyperfinite $\mathrm{II}_{1}$ factor will be of the form

$$
\left\{A_{\alpha_{i}}(S(X, G)) \mid i \in I\right\}
$$

for a particular $G$-space $(X, \mu, G)$ having pure point spectrum, and some uncountable index set $I$.

The organization of our paper runs as follows. For the convenience of the reader we have tried to make the paper as self-contained as possible. Thus the first section contains definitions and general background information on ergodic actions of countable groups and the corresponding von Neumann algebras, and may be skipped by the knowledgeable reader. The second section contains the results on the normalizer of $S(X, G)$ and discusses the Takesaki equivalence relation for the pair $(S(X, G), F(X, G))$. Section 3 discusses the problem of ergodicity and point-spectrum for abelian skew product extensions, emphasizing the relationship between the corresponding von Neumann algebras and crossed product von Neumann algebras by countable abelian automorphism groups. The final section uses the results of $\S 3$ and orbit equivalence to exhibit the uncountable family by pairwise non-inner conjugate Cartan subalgebras of the hyperfinite $\mathrm{II}_{1}$ factor.

1. Ergodic actions of countable groups and the corresponding von Neumann algebras. Let $(X, \mu)$ be a compact Lebesgue space; i.e. $(X, \mu)$ is a standard Borel measure space where $\mu$ is non-atomic, positive, countably additive and $\mu(X)=1$. Let $G$ be a countable discrete group acting on $(X, \mu)$ as a group of Borel isomorphisms so as to leave $\mu$ quasi-invariant; the triple $(X, \mu, G)$ is termed a $G$-space. Throughout the 
following we will be mainly concerned with the case where $G$ acts freely and properly ergodically on $(X, \mu)$ so as to leave the measure $\mu$ invariant.

Let $(X, \mu, G)$ be a space, and let $H$ be a locally compact group. We denote by $Z^{1}((X, G) ; H)$ the one cocycles of $(X, \mu, G)$ with values in $H$, i.e. those Borel maps $\alpha: X \times G \rightarrow H$ such that for all $g_{1}, g_{2} \in G$, $\alpha\left(x, g_{1} g_{2}\right)=\alpha\left(x, g_{1}\right) \alpha\left(x g_{1}, g_{2}\right) \mu$ a.e. Two cocycles $\alpha, \beta \in Z^{1}((X, G) ; H)$ are termed cohomologous, written $\alpha \sim \beta$, if there is a Borel function $b$ : $X \rightarrow H$ such that $\forall g \in G, \alpha(x, g)=b(x)^{-1} \beta(x, g) b(x g) \mu$ a.e. The set of cohomology classes of $Z^{1}((X, G) ; H)$ is denoted by $H^{1}((X, G) ; H)$. We refer to those elements of $Z^{1}((X, G) ; H)$ which are cohomologous to the constant identity cocycle as coboundaries: denote this set by $B^{1}((X, G) ; H)$.

A $G$-space $(Y, \nu, G)$ is said to be a quotient $G$-space of $(X, \mu, G)$ is there exists a $G$-equivariant surjective Borel map $\varphi: X \rightarrow Y$ with $\varphi_{*} \mu=\nu$. Alternatively $(X, \mu, G)$ is called an extension $G$-space of $(Y, \nu, G)$. Let $\left(K, \nu_{K}\right)$ be a separable compact group, and suppose that $\alpha \in$ $Z^{1}((X, G) ; K)$. Define an action of $G$ on $\left(Y \times K, \nu \times \nu_{k}\right)$ by setting $(y, k) g=(y g, k \alpha(y, g))$. It we define $\varphi: X \rightarrow Y$ by $\varphi(y, k)=y$, then $(Y, \nu, G)$ is a quotient $G$-space of $(X, \mu, G)$ with respect to the map $\varphi$. Call $(X, \mu, G)$ a skew-product extension of $(Y, \nu, G)$ by the compact group $K$. Such actions have been extensively studied by Zimmer in [16].

Given a $G$-space $(X, \mu, G)$, there is a natural way of associating to it a von Neumann algebra of operators on a Hilbert space. We follow here the description given in [23, V. 7]. Let $\mathscr{H}$ be the Hilbert space $L^{2}\left(X \times G, \mu \times \nu_{G}\right)$ and $r: X \times G \rightarrow \mathbf{R}^{+}$be the Radon-Nikodym derivative for the action of $G$ on $(X, \mu)$. Construct a unitary representation of $G$ on $\mathscr{H}$ by defining

$$
U_{g_{1}}(f)(x, g)=r\left(x, g_{1}\right)^{1 / 2} f\left(x g_{1}, g_{1}^{-1} g\right), \quad x \in X, g_{1}, g \in G .
$$

Construct a representation of $L^{\infty}(X)$ on $\mathscr{H}$ by defining $T_{\gamma}(f)(x, g)=$ $\gamma(x) f(x, g), \gamma \in L^{\infty}(X), x \in X, g \in G$. Let $F(X, G)$ be the von Neumann algebra generated by $\left\{U_{g} \mid g \in G\right\}$ and $\left\{T_{\gamma} \mid \gamma \in L^{\infty}(X)\right\}$, the socalled group measure von Neumann algebra corresponding to the $G$-space $(X, \mu, G)$. Every element of $F(X, G)$ has a unique Hilbert algebra representation in the form $\sum_{g \in G} T_{\gamma_{g}} U_{g}, \gamma_{g} \in L^{\infty}(X)$. Denote by $S(X, G)$ the von Neumann subalgebra of $F(X, G)$ generated by $\left\{U_{g} \mid g \in G\right\}$, and by $R(X, G)$ the von Neumann subalgebra generated by $\left\{T_{\gamma} \mid \gamma \in L^{\infty}(X)\right\}$. If $(X, \mu, G)$ is a free ergodic $G$-space, $F(X, G)$ will be a factor, and when $\mu$ is $G$-invariant, this factor will be of type $\mathrm{II}_{1}$.

The "crossed-product" construction gives a slightly different construction of a von Neumann algebra corresponding to $(X, \mu, G)$. We briefly discuss this construction here, since dealing with subalgebras 
corresponding to quotient $G$-spaces becomes easier in this context. Let $\mathscr{A}$ be a von Neumann algebra with faithful representation on a Hilbert space $\mathscr{H}_{1}$, and let $G$ be a locally compact group with Haar measure $\gamma_{G}$. Suppose that $g \mapsto \alpha_{g}$ is a strongly continuous homomorphism of $G$ into the group of $*$-automorphisms of $\mathscr{A}$. Define a representation of $\Pi$ of $\mathscr{A}$ on the Hilbert space $L^{2}(G) \otimes \mathscr{H}_{1}=L^{2}\left(G, \mathscr{H}_{1}\right)$ by

$$
\Pi(f)(\zeta)(g)=\alpha_{g}(f) \zeta(g), \quad \zeta \in L^{2}\left(G, \mathscr{H}_{1}\right), f \in \mathscr{A} .
$$

Define a unitary representation of $G$ on $L^{2}\left(G, \mathscr{H}_{1}\right)$ by

$$
u\left(g_{1}\right) \zeta(g)=\zeta\left(g g_{1}\right), \quad g, g_{1} \in G, \zeta \in L^{2}\left(G, \mathscr{H}_{1}\right) .
$$

The von Neumann algebra generated by $\{\Pi(f) \mid f \in \mathscr{A}\}$ and $\{u(g) \mid g \in$ $G\}$ is defined to be the crossed-product of $\mathscr{A}$ by the action $\alpha$ of $G$, written $\mathscr{A} \times{ }_{\alpha} G$. If $G$ is countable and discrete, every element in $\mathscr{A} \times{ }_{\alpha} G$ has a unique "Fourier" expansion in the form $\sum_{g \in G} \Pi\left(f_{g}\right) u(g), f_{g} \in \mathscr{A}$. Let $\mathscr{A}=L^{\infty}(X)$ acting on $L^{2}(X)$ by pointwise multiplication and let the action of $G$ on $L^{\infty}(X)$ be defined by $\alpha_{g}(f)(x)=f(x g)$. Then $L^{\infty}(X) \times{ }_{\alpha} G$ is precisely the commutant of $F(X, G)[23, \mathrm{~V} .7]$, and thus is spatially *-isomorphic to $F(X, G)$ via the unitary involution $J_{X}: L^{2}(X \times G) \rightarrow$ $L^{2}(X \times G)$ defined by $J_{X}(f)(x, g)=r(x, g)^{1 / 2} u\left(x g, g^{-1}\right)$. Indeed $J_{X}\left(U_{g}\right) J_{X}=u(g) \forall g \in G$ and $J_{X} T_{\gamma} J_{X}=\Pi(\gamma) \forall \gamma \in L^{\infty}(X)$.

Using the above constructions we are able to prove the following proposition, which no doubt has been noticed by others:

Proposition 1.1. Let $(Y, \nu, G)$, be an ergodic $G$-space, where $G$ is a countable discrete group and $(Y, \nu)$ is a compact Lebesgue space, and let $(X, \mu, G)$ be an extension $G$-space where $\varphi: X \rightarrow Y$ is the quotient G-map. Then associated to the map $\varphi$ there is a natural injection $\varphi^{*}$ of $F(Y, G)$ into $F(x, G)$.

Proof. Let $m \in F(Y, G)$ and suppose that $m$ has the unique representation $m=\sum_{g \in G} T_{\gamma_{g}} U_{g}, \gamma_{g} \in L^{\infty}(Y), \forall g \in G$, where $m$ is an operator on the Hilbert space $L^{2}(Y \times G)$. There is an obvious injection of $L^{\infty}(Y)$ into $L^{\infty}(X)$ obtained by lifting functions on $Y$ to functions on $X$ via $\varphi$. A natural candidate for $\varphi^{*}(m)$ is therefore the formal sum $\sum_{g \in G} T_{\gamma_{g} \circ \varphi} U_{g}$, and indeed as defined on the generators $\varphi^{*}$ preserves all the arithmetic operations, and is clearly one-to-one. One needs only to check that $\sum_{g \in G} T_{\gamma_{g} \circ \varphi} U_{g}$ is well defined as an element of $B\left(L^{2}(X \times G)\right)$. To do this, it is easier to examine $L^{\infty}(X) \times{ }_{\alpha} G=J_{X} F(X, G) J_{X}$. Since $(Y, \nu, G)$ is ergodic, we can decompose $(X, \mu)$ as a product space $(Y \times D, \nu \times \rho)$, where $D$ is a standard Borel space and $\rho$ is a finite measure on $D$, in such 
a fashion that if $\psi: Y \times D \rightarrow X$ is the Borel isomorphism, then $\varphi_{*}(\mu)=$ $\nu \times \rho$ and $\varphi(\psi(y, d))=y \nu$ a.e. [20]. Thus $L^{2}(X \times G)$ may naturally be regarded as $L^{2}(D) \otimes L^{2}(Y \times G)$. The von Neumann algebra acting on $L^{2}(D) \otimes L^{2}(Y \times G)$ generated by $\left\{\Pi(\gamma \circ \varphi) \mid \gamma \in L^{\infty}(Y)\right\}$ and $\{u(g) \mid$ $g \in G\}$, which we denote by $\mathscr{M}$, is just the tensor product von Neumann algebra $\mathrm{CId} \otimes J_{Y} F_{(Y, G)} J_{Y}$ and hence is clearly *-isomorphic to $F(Y, G)$. Indeed for $m=\sum T_{\gamma_{G}} U_{g} \in F(Y, G), \mathrm{Id}_{L^{2}(D)} \otimes J_{Y} m J_{Y}$ can clearly be identified with $\sum_{g \in G} \Pi\left(\gamma_{g} \circ \varphi\right) u(g) \in J_{X} F_{(X, G)} J_{X}$. This implies that $J_{X}\left(\sum_{g \in G} \Pi\left(\gamma_{g} \circ \varphi\right) u(g)\right) J_{X}=\sum_{g \in G} T_{\gamma_{g} \circ \varphi} U_{g}$ is well defined as an element of $F(X, G)$. Therefore the map $\varphi^{*}$ defined on $F(Y, G)$ by $\varphi^{*}\left(\sum_{g \in G} T_{\gamma_{g}} U_{g}\right)=$ $\sum_{g \in G} T_{\gamma_{g} \circ \varphi} U_{g}$ makes sense, and since

$$
\varphi^{*}(m)=\operatorname{Ad}\left(J_{X}\right)\left(\operatorname{Id}_{L^{2}(D)} \otimes \operatorname{Ad} J_{Y}(m)\right)
$$

is the composition of two *-monomorphisms, $\varphi^{*}$ is itself a $*$-monomorphism of $F(Y, G)$ into $F(X, G)$, as desired. The image of $\varphi^{*}$ under this injection is equal to the von Neumann subalgebra generated by $\left\{T_{\gamma \circ \varphi} \mid \gamma \in L^{\infty}(Y)\right\}$ and $\left\{U_{g} \mid g \in G\right\}$.

We conclude this section with two examples:

ExAmple 1.2. Let $(X, \mu, G)$ be a $G$ space, and let (pt., $1, G)$ be the trivial ergodic action of $G$ on a point. Proposition 1.1 shows that the von Neumann algebra generated by the left regular representation of $G, \mathscr{L}(G)$, injects into $F(X, G)$. The image of $\mathscr{L}(G)$ under this injection is just $S(X, G)$. If $G$ is abelian $S(X, G)$ is *-isomorphic to $L^{\infty}\left(\hat{G}, \nu_{\hat{G}}\right)$ via the Fourier transform.

EXAmple 1.3. Let $(Y, \nu, G)$ be an ergodic $G$ space and let $\beta: Y \times G$ $\rightarrow A$ be an element of $Z^{1}((Y, G), A)$ where $A$ is a separable compact abelian group. Form the skew product $G$-space $\left(Y \times{ }_{\beta} A, \nu \times \nu_{A}, G\right)$. By the proposition $F(Y, G)$ injects naturally into $F\left(Y \times{ }_{\beta} A, G\right)$. Indeed, $F\left(Y \times{ }_{\beta} A, G\right)$ is naturally $*$-isomorphic to a certain crossed product $F(Y, G) \times_{\hat{\beta}} \hat{A}$, as will be detailed in $\S 3$.

2. The normalizer of $S(X, G)$ in $F(X, G)$. Throughout this section we assume that $G$ is a countable abelian group acting freely and ergodically on the compact Lebesgue space $(X, \mu)$ so as to preserve the finite measure $\mu$. As first noticed in [9], in this case both the subalgebras $S(X, G)$ and $R(X, G)$ are maximal abelian in $F(X, G)$. The following 
invariants for maximal abelian subalgebras of factors were first introduced by Dixmier:

Definition 2.1 ([3]). Let $\mathscr{A}$ be a maximal abelian subalgebra in a factor $\mathscr{M}$. Then $\mathscr{A}$ is termed (regular, semi-regular, singular) in $\mathscr{M}$ if the unitaries in $\mathscr{M}$ which normalize $\mathscr{A}$ generate $(\mathscr{M}$, a proper subfactor of $\mathscr{M}$, only $\mathscr{A}$ ) respectively.

It is clear that $R(X, G)$ is always regular maximal abelian in $F(X, G)$. The well-known theorem of Connes, Weiss and Feldman [1] has shown that all regular maximal abelian subalgebras of the hyperfinite $\mathrm{II}_{1}$ factor $\mathscr{R}$ are conjugate by some element of $\operatorname{Aut}(\mathscr{R})$. Therefore, under conditions stated at the beginning of this section, in no sense is the position of the subalgebra $R(X, G)$ in $F(X, G)$ an invariant of the measure-theoretic isomorphism class of the system $(X, \mu, G)$. However, the situation of the maximal abelian subalgebra $S(X, G)$ in $F(X, G)$ has been much less studied. O. Nielsen proved in [16] that if $(X, \mu, G)$ has no non-trivial eigenfunctions, (i.e. has continuous spectrum) then $S(X, G)$ is simple in $F(X, G)$. His result motivated us to study the general case.

Before proceeding to an arbitrary $G$-space, it is illuminating to examine the case where $(X, \mu, G)$ has pure point spectrum, so that up to measure-theoretic isomorphism $(X, \mu)$ is a separable compact abelian group with $G$ embedded as a dense subgroup. Denote this embedding by $\rho: G \rightarrow X$. It is an easy calculation that $\forall \chi \in \hat{X}$, the unitary $T_{\chi}$ normalizes $S(X, G)$; in fact $T_{\chi}^{*} U_{g} T_{\chi}=\chi(\rho(g)) U_{g} \forall g \in G$. As $\left\{T_{\chi} \mid \chi \in \hat{X}\right\}$ and $\left\{U_{g} \mid\right.$ $g \in G\}$ generate $F(X, G)$, the maximal abelian subalgebra $S(X, G)$ is regular in $F(X, G)$.

Indeed, given the embedding $\rho: G \rightarrow X$ there is a dual embedding $\hat{\rho}$ : $\hat{X} \rightarrow \hat{G}$, whose image is dense since $\rho$ is one-to-one, and which is one-to-one since $\operatorname{Im} \rho$ is dense. Therefore one obtains a natural action of the countable abelian group $\hat{X}$ on the compact abelian group $\hat{G}$ given by $\gamma \cdot \chi=\gamma \hat{\rho}(\chi), \chi \in \hat{X}, \gamma \in \hat{G}$; this action gives rise to a group measure factor $F(\hat{G}, \hat{X})$. There is a natural *-isomorphism between $F(X, G)$ and $F(\hat{G}, \hat{X})$ which carries $S(X, G)$ onto $R(\hat{G}, \hat{X})$ and $R(X, G)$ onto $S(\hat{G}, \hat{X})$. To see this, let $\mathscr{F}: L^{2}(X) \otimes L^{2}(G) \rightarrow L^{2}(\hat{X}) \otimes L^{2}(\hat{G})$ be given by $\mathscr{F}_{\hat{X}}^{*} \otimes \mathscr{F}_{G}$, where $\mathscr{F}_{\hat{X}}$ is the Fourier transform carrying $\delta_{\chi} \rightarrow \chi(x), \chi \in \hat{X}$, $x \in X$ and $\mathscr{F}_{G}$ is the Fourier transform sending $\delta_{g} \rightarrow g(\gamma), g \in G, \gamma \in \hat{G}$. Let $J_{\hat{G}}$ be the unitary involution on $L^{2}(\hat{G}) \otimes L^{2}(\hat{X})$ associated to the group measure factor $F(\hat{G}, \hat{X})$. Then $\operatorname{Ad}\left(J_{\hat{G}} \mathscr{F}\right)$ is the desired $*$-isomorphism; a version of this fact was first noted by P. Hahn in [8]. In fact $\operatorname{Ad}\left(J_{\hat{G}} \mathscr{F}\right)\left(T_{\chi}\right)=U_{\chi}, \forall \chi \in \hat{X}$, and $\operatorname{Ad}\left(J_{\hat{G}} \mathscr{F}\right)\left(U_{g}\right)=T_{g}, \forall g \in \hat{G}$, thus 
$\operatorname{Ad}\left(J_{\hat{G}} \mathscr{F}\right)$ maps $S(X, G)$ and $R(X, G)$ and $S(\hat{G}, \hat{X})$, respectively. Now if $\left(X_{1}, \mu_{1}, G_{1}\right)$ is an arbitrary free ergodic $G$-space, a result of Singer ([21], extended to the case $\mu$ quasi-invariant by Zeller-Meier [25]) gives a general formula for all unitary elements of $F\left(X_{1}, G_{1}\right)$ which normalize $R\left(X_{1}, G_{1}\right)$. Using Singer's formula, we can compute the unitaries in $F(\hat{G}, \hat{X})$ which normalize $R(\hat{G}, \hat{X})$ and then pull them back via $\operatorname{Ad}\left(\mathscr{F}^{*} J_{\hat{G}}\right)$ to find those unitaries in $F(X, G)$ which normalize $S(X, G)$. To be specific, we have proved the following generalization of Hahn's remarks in [8]:

Proposition 2.2. Let $G$ be a countable abelian group acting ergodically on the compact Lebesgue space $(X, \mu)$ so as to preserve the finite measure $\mu$, and suppose that the system $(X, \mu, G)$ has pure point spectrum. Then $S(X, G)$ is regular maximal abelian in $F(X, G)$, and any unitary in $F(X, G)$ which normalizes $S(X, G)$ must have the form

$$
\mathscr{F} * J_{\hat{G}}\left(T_{f(\gamma)} \sum_{\chi_{1} \in \hat{X}} T_{\chi_{E_{\chi_{1}}}} U_{\chi_{1}}\right) J_{\hat{G}} \mathscr{F},
$$

where the sets $\left[E_{\chi_{1}} \mid \chi_{1} \in \hat{X}\right\}$ are measurable subset of $\hat{G}$ satisfying (w.r.t. the action of $\hat{X}$ on $\left.\left(\hat{G}, \nu_{\hat{G}}\right)\right)$

$$
\begin{aligned}
& \nu_{\hat{G}}\left(\hat{G}-\underset{\chi_{1} \in \hat{X}}{\bigcup} E_{\chi_{1}}\right)=0 \\
& \nu_{\hat{G}}\left(E_{\chi_{1}} \cap E_{\chi_{2}}\right)=0, \quad \chi_{1} \neq \chi_{2}, \\
& \nu_{\hat{G}}\left(\left(E_{\chi_{1}}\right) \chi_{1} \cap\left(E_{\chi_{2}}\right) \chi_{2}\right)=0, \quad \chi_{1} \neq \chi_{2},
\end{aligned}
$$

and $f: \hat{G} \rightarrow S^{1}$ is any measurable function. Conversely any unitary element in $F(X, G)$ of this form normalizes $S(X, G)$.

In the course of calculating the unitaries in $F(X, G)$ which normalize $S(X, G)$ for more general $G$-spaces $(X, \mu, G)$ we shall need some technical devices of direct integral decomposition due to Takesaki [22]. To be in a positive to use this machinery it is first necessary to represent the pair $(F(X, G), S(X, G))$ on the direct integral Hilbert space $\int_{\Gamma}^{\oplus}\left(L^{2}(X)\right)_{\gamma} d \nu$ (where $\Gamma=\hat{G}, \nu=$ Haar measure on $\Gamma$ ) in such a fashion that $S(X, G)$ is represented as the diagonizable operators $\mathscr{D}=L^{\infty}(\Gamma, \nu)$. We give here a representation constructed by Nielsen [16]. Identify $L^{2}(X) \otimes L^{2}(G)$ with $\int_{\Gamma}^{\oplus}\left(L^{2}(X)\right)_{\gamma} d \nu$ by corresponding to $u(x) \otimes \delta_{g}$ the square integrable vector field $\left(\gamma \rightarrow g^{-1}(\gamma) u(x)\right) \forall g \in G, \forall u \in L^{2}(X)$. Denote this identification operator by $\mathscr{F}$. Let $K$ be the unitary operator mapping $L^{2}(X) \otimes L^{2}(G)$ to itself defined by $K\left(u(x) \otimes \delta_{g}\right)=u(x g) \otimes \delta_{g}$. Then $\operatorname{Ad}\left(\mathscr{F} K^{*}\right)$ is the 
desired representation of $(F(X, G), S(X, G))$ on $\int_{\Gamma}^{\oplus}\left(L^{2}(X)\right)_{\gamma} d \nu$. In particular $\forall g \in G, \operatorname{Ad}\left(\mathscr{F} K^{*}\right)\left(U_{G}\right)$ is the diagonal operator $D_{g}: \gamma \rightarrow g^{-1}(\gamma)$. Let $\Pi$ denote the representation of $F(X, G)^{\prime}=J_{X} F(X, G) J_{X}$ (regarded as an abstract $W^{*}$-algebra) on $\int_{\Gamma}^{\oplus}\left(L^{2}(X)\right)_{\gamma} d \nu$ given by $\operatorname{Ad}\left(\mathscr{F} K^{*}\right)$. Then operators in $\Phi\left(F(X, G)^{\prime}\right)$ will be decomposable elements of $\mathscr{B}\left(\int_{\Gamma}^{\oplus}\left(L^{2}(X)\right)_{\gamma} d \nu\right)$ since $F(X, G)^{\prime} \subset S(X, G)^{\prime}$. Indeed a calculation shows that if $f \in L^{\infty}(X), g \in G$, then $\Pi\left(J_{X} T_{f} U_{g} J_{X}\right)$ is given by the Borel field of operators $\left(\gamma \mapsto \gamma(g) f U^{g}\right)$, where for $g \in G, U^{g}: L^{2}(X) \rightarrow L^{2}(X)$ is the unitary operator defined by $U^{g}(u)(x)=u(x g), u \in L^{2}(X)$, and where for $f \in L^{\infty}(X) f: L^{2}(X) \rightarrow L^{2}(X)$ is defined by pointwise multiplication. Now let $L^{\infty}(X)_{0}$ be a norm-separable $C^{*}$-algebra weakly dense in $L^{\infty}(X)$. Let $\mathscr{U}$ be the $C^{*}$-algebra acting on $L^{2}(X \times G)$ generated by the $T_{f} U_{g}$ where $f \in L^{\infty}(X)_{0}$ and $g \in G$. Then $\mathscr{U}$ is a norm-separable $C^{*}$-algebra weakly dense in $F(X, G)$ and $J_{X} \mathscr{U} J_{X}$ is a norm-separable $C^{*}$-algebra weakly dense in $F(X, G)^{\prime}$. Using results from [22], the representation $\Pi$ restricted to $J_{X} \mathscr{U} J_{X}$ can be decomposed as a direct integral of representations $\Pi=\int_{\Gamma}^{\oplus} \Pi_{\gamma} d \nu$, where each $\Pi_{\gamma}$ represents $J_{X} \mathscr{U} J_{X}$ on $L^{2}(X)_{\gamma}$. The Takesaki equivalence relation for the pair $(F(X, G), S(X, G))$ will be the subset $\left\{\left(\gamma_{1}, \gamma_{2}\right) \in \Gamma \times \Gamma \mid \Pi_{\gamma_{1}}\right.$ is unitarily equivalent to $\left.\Pi_{\gamma_{2}}\right\}$. We refer the reader to [2] for the definition of the Takesaki equivalence relation for a general von Neumann algebra-maximal abelian subalgebra pair $(\mathscr{M}, \mathscr{A})$.

With the above representation $\operatorname{Ad}\left(\mathscr{F} K^{*}\right)$ of $F(X, G), S(X, G)$ in hand we are prepared to prove

THEOREM 2.3. Let $(X, \mu, G)$ be a free ergodic action of the countable discrete abelian group $G$ on the compact Lebesgue space $(X, \mu)$ which preserves the finite measure $\mu$. Then the normalizer of $S(X, G)$ in $F(X, G)$ is the subalgebra $F\left(Y_{d}, G\right)$, where $\left(Y_{d}, \eta, G\right)$ is the maximal quotient action of $(X, \mu, G)$ having pure point spectrum.

Here by 'normalizer' of $S(X, G)$ we mean the von Neumann subalgebra of $F(X, G)$ generated by the unitary elements in $F(X, G)$ normalizing $S(X, G)$. Before proving the theorem we state this immediate

Corollary 2.4. Let $(X, \mu, G)$ be as in Theorem 2.5. Then the maximal abelian subalgebra $S(X, G)$ of $F(X, G)$ is

(1) regular if and only if $(X, \mu, G)$ has pure point spectrum

(2) semi-regular if and only if the maximal quotient action of $(X, \mu, G)$ having pure point spectum is a free action

(3) singular if and only if $(X, \mu, G)$ has no non-trivial point spectrum, i.e. is weak-mixing. 
Statement (3) was first proved by Nielsen using methods which we have extended to the general case. We remark that Theorem 2.4 is very much related to the work of P. Hahn [8], who determined the Takesaki equivalence relation for $(F(X, G), S(X, G))$. However the above formulation, emphasizing the normalizer subalgebra corresponding to the maximal quotient action with pure point spectrum, is new. Since in our proof the structure of the unitaries normalizing $S(X, G)$ is deduced, and the relationship to the results of Singer mentioned in Proposition 2.2 is emphasized, we believe our version is worth repeating.

Proof of Theorem 2.3. Suppose that we are given the representation of $F(X, G)=\mathscr{M}$ on $\int_{\Gamma}^{\oplus}\left(L^{2}(X)\right)_{\gamma} d \nu$ described in the previous paragraphs so that $S(X, G) \cong L^{\infty}(\Gamma, \nu)$ is represented as the diagonizable operators $\mathscr{D}$. Let $U$ be a unitary in $\mathscr{M}$ with

$$
U^{*}(S(X, G)) U=S(X, G) .
$$

Then using a decomposition due to Guichardet [7], there must exist a decomposable unitary operator $T$ given by the Borel field of unitary operators $\gamma \rightarrow T_{\gamma}$ and a $\nu$-measurable map $\theta: \Gamma^{\prime} \rightarrow \Gamma^{\prime}$ where $\Gamma^{\prime}$ is a conull Borel set of $\Gamma$, such that

$$
U=T V
$$

where $V$ is the operator

$$
V(\gamma \rightarrow u(\gamma))=\left(\gamma \rightarrow r(\theta, \gamma)^{-1 / 2} u\left(\theta^{-1} \gamma\right)\right.
$$

(Here $(\gamma \rightarrow u(\gamma))$ represents an arbitrary vector field in $\int_{\Gamma}^{\oplus} L^{2}(X)_{\gamma} d \nu, \theta$ is the map associated to the adjoint action of $U$ on $S(X, G) \cong L^{\infty}(\Gamma)$ by Mackey's point realization theorem, and $r(\theta, \gamma)$ is the Radon-Nikodym derivative $d \nu\left(\theta^{-1}\right) / d \nu$.)

Since $F(X, G)$ is a $\mathrm{II}_{1}$ factor, it has a trace; hence for every $m \in$ $S(X, G), \operatorname{tr}\left(U^{*} m U\right)=\operatorname{tr}(m)$. This implies $r(\theta, \nu) \equiv 1$.

We now let $\Pi$ be the representation of $J_{X} \mathscr{U} J_{X} \subset F(X, G)^{\prime}$ on $\int_{\Gamma}^{\oplus}\left(L^{2}(X)\right)_{\gamma} d \nu$ described above. Recall that $\Pi$ may be decomposed as a direct integral representation $\int_{\Gamma}^{\oplus} \Pi_{\gamma} d \nu$, where the representation $\Pi_{\gamma}$ of $J_{X} \mathscr{U} J_{X}$ on $L^{2}(X)_{\gamma}=L^{2}(X)$ is given by $\Pi_{\gamma}\left(J_{X} t_{f} U_{g} J_{X}\right)=\gamma(g) f U^{g}, \forall f \in$ $L^{\infty}(X)_{0}, \forall g \in G$. Then note that for almost $\gamma \in \Gamma^{\prime}, \Pi_{\gamma}$ is unitarily equivalent to $\Pi_{\theta^{-1}(\gamma)}$. For ease of notation also denote this conull subset of $\Gamma$ by $\Gamma^{\prime}$. Indeed

$$
\Pi_{\gamma} T_{\gamma}=T_{\gamma} \Pi_{\theta^{-1}(\gamma)} \quad \text { for every } \gamma \in \Gamma^{\prime},
$$


where $\gamma \rightarrow T_{\gamma}$ is the decomposition of $T$ into unitary operators mentioned above (see Thm. 1.2 of [22]). Therefore for every $\gamma \in \Gamma^{\prime}, \forall g \in G$ and $\forall f \in L^{\infty}(X)_{0}$,

$$
\gamma(g) f U^{g} T_{\gamma}=T_{\gamma} \theta^{-1}(\gamma)(g) f U^{g}
$$

Taking $g=e$ we obtain

$$
f T_{\gamma}=T_{\gamma} f \quad \forall f \in L^{\infty}(X)_{0} .
$$

Hence $T_{\gamma} \in\left(L^{\infty}(X)_{0}\right)^{\prime}=L^{\infty}(X)$. Therefore suppose that $T_{\gamma}=J_{\gamma}(x) \in$ $L^{\infty}(X)$. Since $T$ is unitary $\left|J_{\gamma}\right|=1$. Now setting $f(x) \equiv 1$ in equation $(*)$, we obtain

$$
\begin{aligned}
\gamma(g) U^{g} J_{\gamma}(x) & =J_{\gamma}(x) \theta^{-1}(\gamma)(g) U^{g} \quad \forall g \in G \\
& \Rightarrow \gamma(g) J_{\gamma}(x g) U^{g}=\theta^{-1}(\gamma)(g) J_{\gamma}(x) U^{g} \quad \forall g \in G \\
& \Rightarrow J_{\gamma}(x g)=\frac{\theta^{-1}(\gamma)}{\gamma}(g) J_{\gamma}(x) \quad \forall g \in G .
\end{aligned}
$$

Therefore $J_{\gamma}$ is an eigenfunction for the action of $G$ on $X$ with eigencharacter $\theta^{-1}(\gamma) \gamma^{-1}$. Since the action of $G$ on $X$ is free, ergodic, and preserves the finite measure $\mu$, there exists an at most countable collection of eigenfunctions for $(X, \mu, G)$ which are orthonormal, and which form a multiplicative group. Call this collection $\left\{f_{\rho}(x) \mid \rho \in \Gamma_{0} \subset \Gamma\right\}$, where $\Gamma_{0}$ is the countable subgroup of $\Gamma=\hat{G}$ corresponding to the eigencharacters.

Set $E_{\rho}=\left\{\gamma \in \Gamma^{\prime} \mid \theta^{-1}(\gamma) \gamma^{-1}=\rho\right\}$. Then $\Gamma^{\prime}=\bigcup_{\rho \in \Gamma_{0}} E_{\rho}$, and

$$
\nu\left(E_{\rho_{1}} \cap E_{\rho_{2}}\right)=0 \text { for } \rho_{1} \neq \rho_{2} \text { in } \Gamma_{0},
$$

and since $\theta^{-1}$ is an invariable transformation, with

$$
\theta^{-1}(\gamma)=\rho \gamma \quad \text { for } \rho \in E_{\rho},
$$

the $E_{\rho}$ must satisfy

$$
\nu\left(E_{\rho_{1}} \cdot \rho_{1} \cap E_{\rho_{2}} \cdot \rho_{2}\right)=0 \text { for } \rho_{1} \neq \rho_{2} \text { in } \Gamma_{0} .
$$

In addition, note that for $\gamma \in E_{\rho}, J_{\gamma}(x)$ and $f_{\rho}(x)$ have the same eigencharacters, thus there must exist $\lambda_{\gamma} \in S^{1}$ satisfying

$$
J_{\gamma}(x)=\lambda_{\gamma} f_{\rho}(x) \text {. }
$$

Let $\lambda$ be the Borel function defined a.e. on $\Gamma^{\prime}$ by $\lambda(\gamma)=\lambda_{\gamma}$.

The above remarks have shown that any unitary $U \in F(X, G)$ having the property that $U^{*}(X, G) U=S(X, G)$ has with respect to the proper representation the form

$$
U=T \cdot V
$$


where $T$ is a decomposable operator whose decomposition is given by

$$
T=\left(\gamma \rightarrow \lambda(\gamma) \sum_{\rho \in \Gamma_{0}} \chi_{E_{\rho}}(\gamma) f_{\rho}\right) \quad\left(f_{\rho} \in L^{\infty}(X) \text { with eigencharacter } \rho\right)
$$

and $V(\gamma \rightarrow u(\gamma))=\left(\gamma \rightarrow u\left(\theta^{-1} \gamma\right)\right)$, where $\theta^{-1}(\gamma)=\gamma \cdot \rho$ for $\gamma \in E_{\rho}$.

One may decompose $U$ even further and note that $T$ is the product $A \cdot T^{\prime}$, where $A$ is the unitary diagonalizable operator $\gamma \rightarrow \lambda(\gamma)$, and $T^{\prime}$ is the decomposable operator

$$
\gamma \rightarrow \sum_{\rho \in \Gamma_{0}} \chi_{E_{\rho}}(\gamma)
$$

Since $A \in L^{\infty}(\Gamma, \nu) \cong S(X, G)$, the operator $U^{\prime}=T^{\prime} \cdot V$ is also in $F(X, G)$ and normalizes $S(X, G)$, so without loss of generality we examine $U^{\prime}$. Our aim is to show that $U^{\prime}$ as an element of $F(X, G)$ is contained in the natural embedding of $F\left(Y_{d}, G\right)$ in $F(X, G)$, where $\left(Y_{d}, \eta, G\right)$ is the maximal quotient action of $(X, \mu, G)$ having pure point spectrum. We do this by actually constructing an element of $F\left(Y_{d}, G\right)$ which maps onto $U^{\prime}$ under $\operatorname{Ad}\left(K \mathscr{F}^{*}\right)$, where recall that $\operatorname{Ad}\left(\mathscr{F} K^{*}\right)$ gives the Takesaki representation of $(F(X, G), S(X, G))$ on $\int_{\Gamma}^{\oplus}\left(L^{2}(X)\right)_{\gamma} d \nu$.

Let $\left\{f_{\rho}(x) \mid \rho \in \Gamma_{0}\right\}$ be the eigenfunctions for $(X, \mu, G)$ chosen as above. Then $T_{f_{\rho}} \in F\left(Y_{d}, G\right)$ for $\rho \in \Gamma_{0}$, and a calculation shows that the operator $\operatorname{Ad}\left(K \mathscr{F}^{*}\right)\left(T_{f_{\rho}}\right)$ sends the vector field $\gamma \rightarrow y(\gamma)$ to the vector field $\gamma \rightarrow f_{\rho}(x) y(\gamma \rho)$. Now let $\mathscr{F}_{\Gamma}^{*}$ be the inverse Fourier transform mapping $L^{2}(G) \rightarrow L^{2}(\Gamma)$ given by $\mathscr{F}_{\Gamma}^{*}\left(\delta_{g}\right)=g^{-1}(\gamma), g \in G, \gamma \in \Gamma$. For every set $E_{\rho}, \rho \in \Gamma_{0}$, associated to the decomposition of the normalizing unitary operator $U^{\prime}$, let

$$
e_{\rho}(g)=\mathscr{F}_{\Gamma}\left(\chi_{E_{\rho}}(\gamma)\right), \quad g \in G, \gamma \in \Gamma .
$$

Since $\chi_{E_{\rho}}(\gamma) \in L^{\infty}(\Gamma, \nu)$, the function $e_{\rho}(g)$ corresponds to a projection in $S(X, G)$, which we denote by $P_{e_{\rho}}$, and thus $P_{e_{\rho}}$ has the Hilbert algebra expansion

$$
P_{e_{\rho}}=\sum_{g \in G} c_{\rho g} U_{g}, \quad \text { with } c_{\rho g} \in \mathbf{C} .
$$

The $\left\{c_{\rho g} \mid g \in G\right\}$ are in fact the coefficients in the Fourier expansion of $\chi_{E_{\rho}}(\gamma)$.

One calculates

$$
\operatorname{Ad} K \mathscr{F} *\left(P_{e_{\rho}} T_{f_{\rho}}\right)(\gamma \rightarrow y(\gamma))=\left(\gamma \rightarrow \chi_{E_{\rho}}(\gamma) f_{\rho}(x) y(\gamma \rho)\right)
$$

where $(\gamma \rightarrow y(\gamma))$ is an arbitrary vector field in $\int_{\Gamma}^{\oplus} L^{2}(X)_{\gamma} d \nu$. Now since

$$
\operatorname{Ad} K \mathscr{F} *\left(U^{\prime}\right)(\gamma \rightarrow y(\gamma))=\left(\gamma \rightarrow \sum_{\rho \in \Gamma_{0}} \chi_{E_{\rho}}(\gamma) f_{\rho}(x) y(\gamma \rho)\right),
$$


it is clear that $\operatorname{Ad} K \mathscr{F}^{*}\left(U^{\prime}\right)=\operatorname{Ad}\left(K \mathscr{F}^{*}\right)\left(\sum_{\rho \in \Gamma_{0}} P_{e_{\rho}} T_{f_{\rho}}\right)$, where $P_{e_{\rho}}$ is a projection in $S(X, G) \forall \rho \in \gamma_{0}$, and $T_{f_{\rho}} \in F\left(Y_{d}, G\right)$ corresponds to an eigenfunction with eigencharacter $\rho, \forall \rho \stackrel{\rho}{\epsilon} \Gamma_{0}$.

Hence $U^{\prime} \in F\left(Y_{d}, G\right)$ as desired, and the decomposition

$$
U^{\prime}=\sum_{\rho \in \Gamma_{0}} P_{e_{\rho}} T_{f_{\rho}}
$$

is just the Hilbert algebra decomposition corresponding to the "pullback" of the corresponding element in $F\left(\hat{G}, \hat{Y}_{d}\right)$ obtained in Proposition 2.2.

Since it is clear that $F\left(Y_{d}, G\right)$ is contained in the von Neumann algebra generated by those unitaries normalizing $S(X, G)$, this completes the proof of the theorem.

REMARK 2.5. The proof of the theorem shows that the Takesaki equivalence relation given by the representation $\operatorname{Ad}\left(K \mathscr{F}^{*}\right)$ of $(F(X, G), S(X, G))$ on $\int_{\Gamma}^{\oplus}\left(L^{2}(X)\right)_{\gamma} d \nu$ is equal to $\{(\gamma, \rho \gamma) \mid \gamma \in \Gamma, \rho \in$ $\left.\Gamma_{0}\right\}$. This was one of Hahn's results in [8]. The equivalence relation will be ergodic in the sense of [11], [5] if and only if $\Gamma_{0}$ is dense in $\Gamma$, hence if and only if the action of $G$ on $\left(Y_{d}, \nu\right)$ is free.

Under the suppositions of the Theorem $F(X, G)$ will be a $\mathrm{II}_{1}$ factor (in fact the hyperfinite $\mathrm{II}_{1}$ factor). Hence $F(X, G)$ has normal faithful conditional expectations onto all of its von Neumann subalgebras. In particular $S(X, G)$ is the image of a normal faithful conditional expectation. Recall that Feldman and Moore in [6] term a maximal abelian subalgebra $\mathscr{A}$ of a factor $\mathscr{M}$ Cartan if it is regular and is the image of a normal faithful conditional expectation. We thus have shown

Corollary 2.6. Let $(X, \mu, G)$ be as in the statement of Theorem 2.4. Then $S(X, G)$ is a Cartan subalgebra of $F(X, G)$ if and only if the system $(X, \mu, G)$ has pure point spectrum.

EXAMPLES. Let the action of $\mathbb{Z}$ on the circle $\left(S^{1}, \nu\right)$ with Haar measure be given by $z \cdot n=\lambda^{n} z$, where $\lambda=e^{2 \pi i \alpha}, \alpha$ irrational. Then $S\left(S^{1}, \mathbb{Z}\right)_{\lambda}$ is a Cartain subalgebra of $F\left(S^{1}, \mathbf{Z}\right)_{\lambda}$.

Let the action of $\mathbf{Z}$ on the torus $\left(S^{1} \times S^{1}, \nu \times \nu\right)$ be generated by the transformation

$$
T: S^{1} \times S^{1} \rightarrow S^{1} \times S^{1} ; \quad T(z, w)=(\lambda z, z w),
$$

where $\lambda$ is as above. Then this action is free and ergodic, and the maximal quotient action of $\left(S^{1} \times S^{1}, \nu \times \nu, Z\right)$ with pure point spectrum is just the system $\left(S^{1}, \nu, \mathbf{Z}\right)$ of the previous paragraph. Therefore $S\left(S^{1} \times S^{1}, \mathbf{Z}\right)$ is 
semiregular in $F\left(S^{1} \times S^{1}, \mathbf{Z}\right)$, with its normalizer equal to the natural injection of $F\left(S^{1}, \mathbf{Z}\right)_{\lambda}$ into $F\left(S^{1} \times S^{1}, Z\right)$.

Let the action of $\mathbf{Z}$ on $\left(\Pi_{-\infty}^{\infty}\{0,1\}_{i}, \Pi_{-\infty}^{\infty} \nu\left(\frac{1}{2}, \frac{1}{2}\right)\right)=\left(X_{\mu}\right)$ be generated by the shift; then $(X, \mu, \mathbf{Z})$ has continuous spectrum so that $S(X, \mathbf{Z})$ is singular in $F(X, \mathbf{Z})$.

Let $(X, \mu, \mathbf{Z})$ be as in the above paragraph and let $\mathbf{Z}$ act on the product space $\left(X \times \mathbf{Z} / 2 \mathbf{Z}, \mu \times \nu\left(\frac{1}{2}, \frac{1}{2}\right)\right)$ by

$$
(x,[i]) n=(x n,[i+n]),
$$

where $[i]$ denotes the image of $i \in \mathbf{Z}$ in $\mathbf{Z} / 2 \mathbf{Z}$. Then the maximal quotient action of $(X \times \mathbf{Z} / 2 \mathbf{Z}, \mu \times \mu, \mathbf{Z})$ having pure point spectrum is given by the transitive action of $\mathbf{Z}$ on $\mathbf{Z} / 2 \mathbf{Z}$. Thus the normalizer of $S(S \times \mathbf{Z} / 2 \mathbf{Z}, \mathbf{Z})$ in $F(X \times \mathbf{Z} / 2 \mathbf{Z}, \mathbf{Z})$ is equal to $F(\mathbf{Z} / 2 \mathbf{Z}, \mathbf{Z})$, which is not a factor, but can be decomposed as a direct integral $\int_{S^{1}}^{\oplus}\left(M_{2}(\mathbf{C})\right) d z$.

3. Abelian skew product extensions of ergodic actions and the corresponding crossed product von Neumann algebras. In this section we use the interconnection between compact abelian skew product extensions and crossed products of finite factors by countable automorphism groups to prove results concerning the point spectrum of compact abelian skew product extensions, when the acting group $G$ is abelian and measure-preserving. These results allow us to solve the inner conjugacy problem for $S(Y, G)$ and $A_{\alpha}(S(Y, G))$ when $(Y, \nu, G)$ has pure point spectrum and $A_{\alpha}$ is the *-automorphism of $F(Y, G)$ corresponding to a one-cocycle $\alpha \in$ $Z^{1}\left((Y, G) ; S^{1}\right)$.

We begin by examining the group-measure von Neumann algebras associated to comapct abelian skew product extensions. Let $(Y, \nu, G)$ be an ergodic $G$-space and suppose that $\beta \in Z^{1}((Y, G) ; A)$ where $A$ is a separable compact abelian group. Form the skew-product $G$-space $\left(Y \times{ }_{\beta} A, \nu \times \nu_{A}, G\right)$. By Proposition $1, F(Y, G)$ injects naturally into $F\left(Y \times{ }_{\beta} A, G\right)$. Indeed we state the following proposition, which is no doubt known to others (for the transformation group $C^{*}$-algebra case, see [19, Theorem 1.5.7]. We keep the notation of $\$ 1$ concerning crossed products.

Proposition 3.1. Let $(Y, \nu, G)$ be an ergodic $G$-space, and let $\beta$ : $Y \times G \rightarrow A$ be a cocycle of $(Y, \nu, G)$ into a separable compact abelian group A. Then there exists an action $\hat{\beta}: \hat{A} \rightarrow \operatorname{Aut}(F(Y, G))$ such that $F(Y, G) \times{ }_{\beta} \hat{A}$ is *-isomorphic to $F\left(Y \times{ }_{\beta} A, G\right)$, and under this *-isomorphism $\Pi(F(Y, G))$ is carried onto $\phi^{*}(F(Y, G))$, where $\phi: Y \times A \rightarrow Y$ is projection in the first variable. 
We leave it to the reader to verify that the action $\hat{\beta}$ of $\hat{A}$ on $F(Y, G)$ is given by

$$
\hat{\beta}(x)\left(\sum_{g \in G} T_{\gamma_{g}} U_{g}\right)=\sum_{g \in G} T_{\chi(\beta(y, g)) \gamma_{g}} U_{g}, \quad \chi \in \hat{A} .
$$

For any $\alpha \in Z^{1}\left((Y, G) ; S^{1}\right)$, let $A_{\alpha}$ denote the $*$-isomorphism of $F(Y, G)$ defined by

$$
A_{\alpha}\left(\sum_{g \in G} T_{\gamma_{g}} U_{g}\right)=\sum_{g \in G} T_{\alpha(y, g)) \gamma_{g}} U_{g}
$$

then clearly $\hat{\beta}(\chi)=A_{\chi \circ \beta^{\prime}} \forall \chi \in \hat{A}$. Note that for $\alpha \in Z^{1}\left((Y, G) ; S^{1}\right), A_{\alpha}$ is inner if and only if $\alpha \in B\left((Y, G) ; S^{1}\right)([21])$.

Thus when $A$ is a separable compact abelian group, the von Neumann algebra $F\left(Y \times{ }_{\beta} A, G\right)$ can be regarded in a natural way as the crossed product of $F(Y, G)$ by a countable group (isomorphic to $\hat{A}$ ) of $*$-isomorphisms each of the form $A_{\alpha}$ for some $\alpha \in Z^{1}\left((Y, G) ; S^{1}\right)$. It is very useful to have this decomposition, since crossed products of finite factors by countable automorphism groups have been extensively studied by Nakumura and Takeda in [14], [15]; several of their results will prove very important to us.

For the remainder of this section, let $(Y, \nu, G)$ be a free ergodic $G$ space, where $G$ preserves the finite meausre $\nu$. We want to restrict our study to compact abelian skew product extensions corresponding to minimal cocycyles. If $\alpha \in Z^{1}((Y, G) ; K)$ for some separable compact group $K$, denote by $K_{\alpha}$ the closed subgroup of $K$ generated by $\{\alpha(y, g) \mid$ $y \in Y, g \in G\}$. Recall from [26] that $\alpha$ is said to be minimal if there is no $\beta \in Z^{1}((Y, G) ; K), \beta \sim \alpha$, with $K_{\beta} \subsetneq K_{\alpha}$. The following proposition provides a test for minimality of a given cocycle with values in a compact abelian group:

Proposition 3.2. Let $(Y, \nu, G)$ be a free ergodic $G$ space, where $G$ preserves the finite measure $\nu$. Let $\alpha \in Z^{1}((Y, G) ; A)$ where $A$ is a separable compact abelian group. Let $B_{\alpha}$ be the subgroup of $\hat{A}$ defined by $B_{\alpha}=\{\chi \in$ $\left.\hat{A} \mid \chi \circ \alpha \in B^{1}\left((Y, G) ; S^{1}\right)\right\}$. Then if $\beta$ is any minimal cocycle cohomologous to $\alpha, A_{\beta}=B_{\alpha}^{\perp}=\left\{a \in A \mid \chi(a)=1 \forall \chi \in B_{\alpha}\right\}$.

Proof. By a result of Zimmer [26, Cor. 3.8], there exists a closed subgroup $A_{m}$ of $A$ and a minimal cocycle $\beta \sim \alpha$ with $A_{m}=A_{\beta}$. The same proof shows that $A_{m}$ is independent of the minimal cocycle $\beta$ chosen, since $A$ is abelian. It is easily checked that $B_{\alpha}$ as defined in the statement of the proposition is a subgroup. We prove that $B_{\alpha}=\left(A_{m}\right)^{\perp}$, where 
$\left(A_{m}\right)^{\perp}=\left\{\chi \in \hat{A} \mid \chi(a)=1 \forall a \in A_{m}\right\}$. As $\left(A_{m}^{\perp}\right)^{\perp}=A_{m}$, the desired result will follow.

We first note that $\left(A_{m}\right)^{\perp} \subset B_{\alpha}$, for suppose that $\chi \in\left(A_{m}\right)^{\perp}$. Then $\chi(\beta(y, g)) \equiv 1$. Since $\beta \sim \alpha$, there exists a Borel $f: Y \rightarrow A$ with $f(y g) \beta(y, g) f(y)^{-1}=\alpha(y, g)$. Therefore,

$$
\begin{aligned}
\chi(\alpha(y, g)) & =\chi(f(y g) \beta(y, g) f(y))^{-1} \\
& =\chi(f(y g)) \chi(\beta(y, g)) \chi(f(y))^{-1}=\chi(f(y g)) \chi(f(y))^{-1} \\
& \Rightarrow \chi \cdot \alpha \text { is a coboundary, } \\
& \therefore \chi \in B_{\alpha} .
\end{aligned}
$$

Now suppose that $\chi \in B_{\alpha}$, so that $\chi \cdot \alpha$ is a coboundary. Then choosing a minimal cocycle $\beta$ with $\beta \sim \alpha, \chi \circ \beta$ will be a coboundary as well, since $\chi$ is a homomorphism. Hence there exists a Borel function $b$ : $Y \rightarrow S^{1}$ satisfying

$$
b(y g) b(y)^{-1}=\chi(\beta(y, g)) \quad \nu \text { a.e., } \quad \forall g \in G .
$$

Examine the following function defined on $Y \times A_{m} ; h(y, z)=b(y) \overline{\chi(z)}$. Note that $h$ is $G$-invariant with respect to the skew product action of $G$ on $\left(Y \times{ }_{\beta} A_{m}, \nu \times \nu_{A_{m}}\right)$, since

$$
\begin{aligned}
h((y, z) g) & =h(y g, z \beta(y, g)) \\
& =b(y g) \bar{\chi}(\beta(y, g)) \overline{\chi(z)}=b(y) \overline{\chi(z)} .
\end{aligned}
$$

But since $\beta$ was chosen minimal with $A_{m}=A_{\beta}$, by ([26, Cor. 3.8]) $\left(Y \times A_{m}, \nu \times \gamma_{A_{m}}, G\right)$ is ergodic, thus $h(y, z)$ must be $\nu \times \nu_{A_{m}}$ a.e. constant. Therefore $b(y)$ is $\nu$ a.e. constant and $\chi$ is a constant function on $A_{m}$ which implies that $\chi \in\left(A_{m}\right)^{\perp}$, as desired.

Proposition 3.2 has several corollaries, the first of which is immediate upon taking $A=S^{1}$.

Corollary 3.3. Let $(Y, \nu, G)$ be as in the statement of 3.2, and let $\alpha \in Z^{1}\left((Y, G) ; S^{1}\right)$. Let $n$ be the least positive integer such that $\alpha^{n}(y, g)=$ $\alpha(y, g))^{n}$ is a coboundary or $\infty$ is no such $n$ exists. Then $\alpha$ is cohomologous to a minimal cocycle $\beta$ taking on values in $D_{n}$, where $D_{n}$ represents the nth roots of unity for $n$ finite and $D_{\infty}=S^{1}$. Furthermore $\left(Y \times_{\beta} D_{n}, \nu \times \nu_{D_{n}}, G\right)$ is ergodic.

Corollary 3.4. Let $(Y, \nu, G)$ be as in 3.2 and let $\alpha \in Z^{1}((Y, G) ; A)$, where $A$ is compact separable abelian. Then $F\left(Y \times{ }_{\alpha} A, G\right)$ is a factor if and only if $B_{\alpha}$ is equal to the identity subgroup. 
Proof. By Zimmer's results quoted above, $\left(Y \times{ }_{\alpha} A, \nu \times \nu_{A}, G\right)$ is ergodic if and only if $\alpha$ is minimal and $A_{\alpha}=A$. Thus by Proposition 3.2 $\left(Y \times{ }_{\alpha} A, \nu \times \nu_{A}, G\right)$ is ergodic iff $B_{\alpha}=\{e\}$. The result then follows from ([23], Cor. V. 7.8).

We thus may restrict ourselves to minimal cocycles when examining ergodic compact abelian skew product extensions. We now investigate the conditions under which such an ergodic extension $\left(Y \times{ }_{\beta} A, \nu \times \nu_{A}, G\right)$ has point structure distinct from that of the quotient action $(Y, \nu, G)$. By using von Neumann algebra techniques, we can prove the following theorem, which in the case where $A=S^{1}$ and $G=\mathbf{Z}$ was first proved by Anzai [27]:

THEOREM 3.5. Let $G$ be a countable discrete abelian group acting freely and ergodically on the compact Lebesgue space $(Y, \nu)$ so as to preserve the finite measure $\nu$. Let $\beta \in Z^{1}((Y, G) ; A)$ be minimal, where $A$ is a separable compact abelian group and $K_{\beta}=A$. Then the skew product ergodic $G$-space $\left(Y \times_{\beta} A, \nu \times \nu_{A}, G\right)$ has point spectrum distinct from the quotient $G$-space $(Y, \nu, G)$ if and only if there exist non-trivial $\gamma \in \hat{G}, \chi \in \hat{A}$, and a Borel function $m: Y \rightarrow S^{1}$ such that

$$
\gamma(g) m(y g) m(y)^{-1}=\chi(\beta(y, g)) \quad \nu \text { a.e., } \quad \forall g \in G
$$

i.e. if and only if $\exists \chi \in \hat{A}$ such that $\chi \circ \beta$ is cohomologous to a cocycle "restricted" from $G$.

Proof. The proof of the theorem uses the interplay between crossedproduct von Neumann algebras and their corresponding generating skew-product dynamical systems. Let $F\left(Y \times{ }_{\beta} A, G\right)$ be the group measure von Neumann algebra corresponding to the system $\left(Y \times_{\beta} A, \nu \times \nu_{A}, G\right)$. Then recall that the finite factor $F\left(Y \times{ }_{\beta} A, G\right)$ is naturally $*$-isomorphic to the crossed product von Neumann algebra $F(Y, G) \times_{\beta} \hat{A}$. Let $e_{\gamma}(y, z)$ be an eigenfunction for $\left(Y \times_{\beta} A, \nu \times \nu_{A}, G\right)$ with a eigencharacter $\gamma$. Then note that

$$
T_{e_{\gamma}} U_{g} T_{e_{\gamma}}^{*}=\overline{\gamma(g)} U_{g} \quad \forall g \in G
$$

and

$$
T_{e_{\gamma}} T_{m} T_{e_{\gamma}}^{*}=T_{m} \text { for } m \in \phi^{*}\left(L^{\infty}(Y)\right) .
$$

Hence $T_{e_{\gamma}}$ is a unitary element which normalizes $\phi^{*}(F(Y, G))$ in $F\left(Y \times{ }_{\beta} A, G\right)$. By a result of Nakamura and Takeda [15], if $\mathscr{M}$ is a finite factor and $D$ is a countable group of outer automorphisms of $\mathscr{M}$, then any 
unitary element $W$ in $\mathscr{M} \times D$ which normalizes $\Pi(\mathscr{M})$ must be of the form $\Pi(U) u(d)$ form some $d \in D$ and some unitary $U \in \mathscr{M}$. By applying Proposition 3.1 and using this result, we see that $T_{e_{\gamma}}$ must be of the form $T_{e_{\gamma}}=\varphi^{*}(U) T_{f_{\chi}}$ for some $\chi \in \hat{A}$ and unitary $U \in F(Y, G)$ where $f_{\chi}(y, z)$ $=\chi(z)$. Then $\phi^{*}(U)=T_{e_{\gamma}} T_{f_{\chi}}$, so that $\phi^{*}(U) \in R\left(Y \times_{\beta} A, G\right) \cap$ $\phi^{*}(F(Y, G))=\phi^{*}(R(Y, G))$. So there must exist $m(y) \in L^{\infty}(Y),|m(y)|$ $=1$ a.e. with $e_{\gamma}(y, z)=m(y) f_{\chi}(y, z)$, for some $\chi \in \hat{A}$. It follows that

$$
e_{\gamma}(y g, \beta(y, g) z)=m(y g) f_{\chi}(y g, z \beta(y, g)) \text {. }
$$

But

$$
e_{\gamma}(y g, \beta(y, g) z)=e_{\gamma}((y, z) g)=\gamma(g) e_{\gamma}(y, z)
$$

hence

$$
\begin{aligned}
\gamma(g) e_{\gamma}(y, z) & =m(y g) \chi(z) \chi(\beta(y, g)) \\
& \Rightarrow \gamma(g)=m(y) m(y g)^{-1} \chi(\beta(y, g)) \\
& \Rightarrow \gamma(g) m(y g) m^{-1}=\chi(\beta(y, g)) .
\end{aligned}
$$

This completes the proof of the theorem.

Remark 3.6. Note that $\{\chi \in \hat{A} \mid \chi$ satisfies (*) for some $\gamma \in G$ and some Borel $\left.m: Y \rightarrow S^{1}\right\}$ will be a subgroup of $\hat{A}$, which we denote by $E$. The proof of the theorem shows that the von Neumann algebra of $F\left(Y \times{ }_{\beta} A, G\right)$ generated by $\varphi^{*}(F(Y, G))$ and $\left\{T_{e_{\gamma}} \mid e_{\gamma}\right.$ is an eigenfunction for $\left.\left(Y \times{ }_{\beta} A, \nu \times \nu_{A}, G\right)\right\}$ will be *-isomorphic to the subalgebra of $F(Y, G) \times{ }_{\beta} \hat{A}$ generated by $\Pi(F(Y, G))$ and $\{u(\chi) \mid \chi \in E\}$, under the natural $*$-isomorphism defined by Proposition 3.1. We denote this subalgebra by $F(Y, G) \times_{\hat{\beta}} E$. In fact, under this *-isomorphism, $F(Y, G) \times_{\hat{\beta}} E$ has a natural group measure-structure, as the following statement shows:

Claim 3.7. Under the *-isomorphism defined in Proposition 3.1, $F(Y, G) \times_{\hat{\beta}} E$ is naturally $*$-isomorphic to $F(X, G)$ where $(X, \mu, G)$ is the quotient action of $\left(Y \times{ }_{\beta} A, \nu \times \nu_{A}, G\right)$ defined by the skew product $\left(Y \times{ }_{\beta} A / E^{\perp}, \nu \times \tilde{\nu}_{A}, G\right)$ where the quotient $G$-map $\varphi^{\prime}: Y \times A \rightarrow Y \times$ $A / E^{\perp}$ is defined by $\varphi^{\prime}(y, z)=(y, \tilde{z})$.

Proof. The proof is nothing more than an application of the result from the Pontryagin duality theory that $(\widehat{A / C})=C^{\perp}$ for any closed subgroup $C$ of $A$. Let $\varphi^{\prime \prime}: Y \times A / E^{\perp} \rightarrow Y$ be the quotient $G$-map defined by projection in the first variable. Note that $\varphi^{\prime \prime} \circ \varphi^{\prime}=\varphi$. It is clear that $\left\{f_{\chi}(y, \bar{z})=\chi(\bar{z}) \mid \chi \in\left(\widehat{A / E^{\perp}}\right)\right\}$ together with $\varphi^{\prime \prime}\left(L^{\infty}(Y)\right)$ generates 
$L^{\infty}\left(Y \times A / E^{\perp}\right)$. Hence $F\left(Y \times A / E^{\perp}, G\right)$ is generated by $\left(\varphi^{\prime \prime}\right)^{*}(F(Y, G))$ and $\left\{T_{f_{x}} \mid \chi \in A / E^{\perp}\right\}$. Consequently $\varphi^{\prime *}\left(F\left(Y \times A / E^{\perp}, G\right)\right)$ is generated by $\varphi^{\prime *} \varphi^{\prime *}(F(Y, G))=\varphi^{*}(F(Y, G))$ and $\left\{T_{f_{x \circ \varphi^{\prime}}} \mid \chi \in\left(\overline{A / E^{\perp}}\right)\right\}$. But by Pontryagin duality theory

$$
\left\{f_{\chi} \circ \varphi^{\prime} \mid \chi \in\left(\widehat{A / E^{\perp}}\right)\right\}=\left\{f_{\chi} \mid \chi \in\left(E^{\perp}\right)^{\perp}=E\right\} .
$$

Therefore $\left(\varphi^{\prime}\right)^{*}\left(F\left(Y \times{ }_{\beta} A / E^{\perp}, G\right)\right)$ is equal to the von Neumann subalgebra of $F\left(Y \times{ }_{\beta} A, G\right)$ generated by $\varphi^{*}(F(Y, G))$ and $\left\{T_{f_{\chi}} \mid \chi \in E\right\}$, and hence is a $*$-isomorphic to $F(Y, G) \times{ }_{\hat{\beta}} E$, as desired.

We are now in a positive to determine under which conditions $A_{\alpha}(S(Y, G))$ is inner conjugate to $S(Y, G)$ when $(Y, \nu, G)$ is a free ergodic $G$-space with pure point spectrum and $\alpha: Y \times G \rightarrow S^{1}$ a 1-cocycle. Clearly it is sufficient that $\alpha$ be a coboundary, since in this case $A_{\alpha}$ will itself be an inner automorphism, but this is not necessary condition. Indeed choose $\gamma \in \hat{G}$ such that $\gamma$ is not an eigencharacter for $(Y, \nu, G)$. Defining $\gamma(y, g)=\gamma(g), A_{\gamma}$ will not be an inner automorphism since $\gamma$ is not a coboundary. In this case, however, it is clear by inspection that

$$
A_{\gamma}(S(Y, G))=S(Y, G) \text {. }
$$

Our results show that, modulo multiplication by a coboundary, in order that $A_{\alpha}(S)$ be inner conjugate to $S, \alpha$ must be a cocycle of the above form:

THEOREM 3.8. Let $(Y, \nu, G)$ be a free ergodic action of the countable abelian group $G$ which preserves the finite measure $\nu$ and which has pure point spectrum. Let $\alpha: Y \times G \rightarrow S^{1}$ be a Borel 1-cocycle. Then $A_{\alpha}(S(Y, G))$ is inner conjugate to $S(Y, G)$ if and only if $\alpha$ is cohomologous to a cocycle of the form $\gamma(x, g)=\gamma(g)$ for some $\gamma \in \hat{G}$.

Proof. Note that if $\beta$ is cohomologous to $\alpha, A_{\beta}(S(Y, G))$ is inner conjugate to $A_{\alpha}(S(Y, G))$, so that without loss of generality we may assume $\alpha$ minimal taking on values in a closed subgroup $D_{m} \subset S^{1}$ with $K_{\alpha}=D_{m}, m \in\{1,2, \ldots, \infty\} ;$ recall $D_{\infty}=S^{1}$.

Suppose that $A_{\alpha}(S(Y, G))$ is inner conjugate to $S(Y, G)$. Then there exists $W \in F(Y, G)$ with

$$
W S(Y, G) W^{*}=A_{\alpha}(S(Y, G)) \text {. }
$$

We now examine the extension action $\left(Y \times{ }_{\alpha} D_{m}, \nu \times v_{D_{m}}, G\right)=$ $(X, \mu, G)$, where $(y, d) g=(y g, d \alpha(y, g))$. Since $\alpha$ is minimal with $K_{\alpha}=$ $D_{m},(X, \mu, G)$ is ergodic. Let $\varphi: X \rightarrow Y$ be the quotient $G$-map given by projection in the first variable. We then can embed $F(Y, G)$ as a subfactor of $F(X, G)$ via the map $\varphi^{*}$. Note that $\alpha(\varphi(x), g)$ is a coboundary in 
$Z^{1}\left((X, G) ; S^{1}\right)$, i.e. there exists a Borel map $f_{\alpha}: X \rightarrow S^{1}$ such that $f_{\alpha}(x g) f_{\alpha}(x)^{-1}=\alpha(\varphi(x), g)$; to see this just define $f_{\alpha}(y, d)=d$. Hence $\varphi^{*}(S(Y, G))$ and $\varphi^{*}\left(A_{\alpha}(S(Y, G))\right)$ are inner conjugate in $F(X, G)$ by Ad $U$, where $U=T_{\tilde{f}_{\alpha}}$. Denoting the subalgebra $S(Y, G)$ by $S$, one obtains

$$
\begin{aligned}
U^{*} \varphi^{*}(W) \varphi^{*}(S) \varphi^{*}\left(W^{*}\right) U & =U^{*} \varphi^{*}\left(W S W^{*}\right) U=U^{*} \varphi^{*}\left(A_{\alpha}(S)\right) U \\
& =\varphi^{*}(S)=S(X, G) .
\end{aligned}
$$

Therefore $\operatorname{Ad}\left(U^{*} \varphi^{*}(W)\right)$ normalizes the maximal abelian subalgebra $S(X, G)$ in $F(X, G)$. But then by Theorem $2.3, U^{*} \varphi^{*}(W)$ is contained in $F(M, G)$ where $(M, G)$ is the maximal quotient action of $(X, \mu, G)$ having pure point spectrum. Note that $(M, G)$ is an extension action of $(Y, \nu, G)$, so that $F(Y, G)$ naturally embeds in $F(M, G)$. Since $\varphi^{*}(W) \in$ $\varphi^{*}(F(Y, G)) \subset F(M, G)$, we obtain $U \in F(M, G)$. But $U^{*}=T_{f_{\alpha}}$ which together with $F(Y, G)$ generates $F(X, G)$. Therefore $F(M, G)=F(X, G)$, which implies that $(X, \mu, G)$ has pure point spectrum. By Remark 3.7 this implies that there exists some $\gamma \in \hat{G}$ with $\alpha(y, g)$ cohomologous to $\gamma(y, g)=\gamma(g)$ in $Z^{1}\left((Y, G) ; S^{1}\right)$. This proves $\Rightarrow$; the other direction is clear from our remarks preceding the theorem.

4. An uncountable family of non-inner conjugate cartan subalgebras in the hyperfinite $\mathrm{II}_{1}$ factor. As mentioned in the introduction, Feldman and Moore, using Connes' results on the simplicity of $\operatorname{Out}(\mathscr{R})=$ $\operatorname{Aut}(\mathscr{R}) / \operatorname{Inn}(\mathscr{R})$, proved in [6] that the hyperfinite $\mathrm{II}_{1}$ factor $\mathscr{R}$ must contain at least two Cartan subalgebras which are not inner conjugate to one another. (Actually, in [13], G. Muravera had constructed two non-inner conjugate regular (hence Cartan) subalgebras of $\mathscr{R}$ ). In a review of [17], the referee noted that by using Connes', Feldman's and Weiss's results on the uniqueness up to outer conjugacy of the Cartan subalgebra in $\mathscr{R}$ in conjunction with the methods of [6] one can easily see that the set of inner conjugacy classes of Cartan subalgebras in $\mathscr{R}$ is uncountable. In this section our aim is to apply the methods developed up to this point to exhibit an uncountable family of Cartan subalgebras in the hyperfinite II $_{1}$ factor, no two of which are inner conjugate. This family will be contained as a sub-family of $\left\{A_{\alpha}\left(S(Y, G) \mid \alpha \in Z^{1}\left((Y, G) ; S^{1}\right)\right\}\right.$ for some free ergodic $G$-space $(Y, \nu, G)$ where $G$ is a countable abelian group, preserves the finite measure $\mu$, and the system $(Y, \nu, G)$ has pure point spectrum. We introduce the following easy

Claim 4.1. Let $(Y, \nu, G)$ be a free ergodic $G$ space, and let $\alpha, \beta \in$ $Z^{1}\left((Y, G) ; S^{1}\right)$. Then $A_{\alpha}(S(Y, G))$ is inner conjugate to $A_{\beta}(S(Y, G))$ if and only if $S(Y, G)$ is inner conjugate to $A_{\alpha^{-1} \beta}(S(Y, G))$. 
Proof. Note that for $U$ unitary in $F(Y, G)$,

$$
\begin{aligned}
& U(S(Y, G)) U^{*}=A_{\alpha^{-1} \beta}(S(Y, G)) \\
& \quad \Leftrightarrow A_{\alpha}\left(U S(Y, G) U^{*}\right)=A_{\alpha}\left(A_{\alpha^{-1} \beta}(S(Y, G))\right) \\
& \quad \Leftrightarrow A_{\alpha}(U) A_{\alpha}(S(Y, G)) A_{\alpha}(U)^{*}=A_{\beta}(S(Y, G)) .
\end{aligned}
$$

Since $A_{\alpha}(U)$ is a unitary element of $F(Y, G)$, the proof is complete.

In what follows, results on the structure of $H^{1}\left((Y, G) ; S^{1}\right)$ will be used often, where $(Y, \nu, G)$ is a free ergodic $G$-space, $G$ is countable abelian, and $\nu$ is finite and $G$-invariant. These results use the idea of orbit equivalence; so we briefly review notions that will be used:

Definition 4.3. Let $\left(X_{1}, \mu_{1}, G_{1}\right)$ and $\left(X_{2}, \mu_{2}, G_{2}\right)$ be free ergodic $G_{1}$ and $G_{2}$ spaces respectively. A Borel map $\Phi: X_{1} \rightarrow X_{2}$ is called an orbit equivalence of $\left(X_{1}, \mu_{1}, G_{1}\right)$ and $\left(X_{2}, \mu_{2}, G_{2}\right)$ if $\Phi$ is a Borel isomorphism with $\Phi_{*}\left(\mu_{2}\right)$ equivalent to $\mu_{1}$ and if $\left\{\Phi\left(x G_{1}\right)\right\}=\left\{\Phi(x) G_{2}\right\}$ for almost every $x \in X_{1}$.

Any such orbit equivalence defines a cocycle $\varphi: X_{1} \times G_{1} \rightarrow G_{2}$ by

$$
\Phi(x g)=\Phi(x) \varphi(x, g) \text {. }
$$

The map $\Lambda: X_{1} \times G_{1} \rightarrow X_{2} \times G_{2}$ given by $\Lambda(x, g)=(\Phi(x), \varphi(x, g))$ will define an isomorphism of ergodic measure groupoids [see [18] for definitions and technical results] and $\Lambda^{*}: L^{2}\left(X_{2} \times G_{2}\right) \rightarrow L^{2}\left(X_{1} \times G_{1}\right)$ gives a spatial isomorphism of $F\left(X_{2}, G_{2}\right)$ and $F\left(X_{1}, G_{1}\right)$. Indeed, the map $\Lambda$ associated to the orbit equivalence defines a one-to-one correspondence between $Z^{1}\left(\left(X_{2}, G_{2}\right) ; L\right)$ and $Z^{1}\left(\left(X_{1}, G_{1}\right) ; L\right)$ for any locally compact second countable group $L$, and this correspondence carries $B^{1}\left(\left(X_{2}, G_{2}\right) ; L\right)$ onto $B^{1}\left(\left(X_{1}, G_{1}\right) ; L\right)$. One thus obtains an equivalnce between $H^{1}\left(\left(X_{2}, G_{2}\right) ; L\right)$ and $H^{1}\left(\left(X_{1}, G_{1}\right) ; L\right)$, and when $L$ is abelian this equivalence is an isomorphism of groups. Therefore it makes sense to refer to $H^{1}(\mathscr{G} ; L)$, where $\mathscr{G}$ represents the orbit equivalence class (or ergodic equivalence relation-see [5], [18] for this point of view) associated to a free ergodic group action.

When $G_{1}$ and $G_{2}$ are abelian, acting freely and ergodically on the compact Lebesgue spaces $\left(X_{1}, \mu_{1}\right),\left(X_{2}, \mu_{2}\right)$ so as to preserve the finite measures $\mu_{1}$ and $\mu_{2}$ respectively, a deep result due to Dye [4] shows that $\left(X_{1}, \mu_{1}, G_{1}\right)$ and $\left(X_{1}, \mu_{2}, G_{2}\right)$ are orbit equivalent. Indeed the results of Connes, Feldman and Weiss cited earlier show that any two free ergodic measure preserving amenable (see [1]) dynamical systems are orbit equivalence (again assuming the groups to be countable discrete), and give rise to the hyperfinite $\mathrm{II}_{1}$ factor via the group measure construction. 
The above remarks show that there are many different ways to go about studying $H^{1}\left(\mathscr{F} ; S^{1}\right)$ where $\mathscr{F}$ (the hyperfinite $I I_{1}$ ergodic groupoid) represents the orbit equivalence class of actions corresponding to the hyperfinite $\mathrm{II}_{1}$ factor. From this point of view, the results of Westman [24] that $H^{1}\left(\mathscr{F} ; S^{1}\right)$ contains as a subgroup every countable discrete abelian group and every compact second countable abelian group, seem very natural.

EXAMPLE 4.3. We briefly discuss a method of showing that $H^{1}\left(\mathscr{F} ; S^{1}\right)$ contains $A$ as a subgroup, where $A$ is any second countable compact abelian group. Let $\hat{A}=D$ which is a countable discrete abelian group. Form the space $\Pi_{d \in D}\{0,1\}_{d}=X$, giving $X$ the product measure $\Pi_{d \in D}\left(\mu_{1 / 2}\right)=\mu$, where $\mu_{1 / 2}$ assigns measure $1 / 2$ to each point in $\{0,1\}$. We write each $x \in X$ as $\left\{x_{d}\right\}_{d \in D}, x_{d} \in\{0,1\}$, and let $D$ act as a group of automorphisms of $(X, \mu)$ by the $D$-shift:

$$
\left(x \cdot d_{1}\right)_{d}=x_{d_{1} d}, \quad d \in D .
$$

The reader is referred to [12] where it is shown in a more general setting that $D$ acts freely, ergodically, and in a measure-preserving fashion on $(X, \mu)$, and that the system $(X, \mu, D)$ has continuous spectrum: i.e. if there exists $f \in L^{2}(X)$ and $\rho \in \hat{D}$ with $f(x d)=\rho(d) f(x)$ a.e., then $f$ is a constant function and $\rho \equiv 1$.

Therefore the group $A=\hat{D}$ embeds into $Z^{1}\left((X, D) ; S^{1}\right)=Z^{1}\left(\mathscr{F} ; S^{1}\right)$ via $\rho(x, d)=\rho(d)$ for $\rho \in A, d \in d$, and $x \in X$, with $\rho_{1}(x, d) \sim \rho_{2}(x, d)$ if and only if $\rho_{1}=\rho_{2}$. This gives the desired embedding of $A$ into $H^{1}\left(\mathscr{F} ; S^{1}\right)$.

Now let $Y=\Pi_{i \in \mathbf{N}}\{0,1\}_{l}$, with product measure $\nu=\Pi_{i \in \mathbf{N}}\left(\nu_{1 / 2}\right)_{i}$, and let $G=\oplus_{i \in N}\{0,1\}_{i}$. Both $G$ and $Y$ have topological group structures defined by coordinate wise addition mod2. It is well-known that $G$ embeds as a dense subgroup of $Y$, and that the $G$-space $(Y, \nu, G)$ defined by this embedding is free, ergodic, measure-preserving, and his pure spectrum. Therefore $F(Y, G)$ is the hyperfinite $\mathrm{II}_{1}$ factor and $S(Y, G)$ is a Cartan subalgebra in $F(Y, G)$. Also $\hat{G}$ is isomorphic to $Y$ so that every element of $\hat{G}$ has order at most 2 .

Let $A_{3}$ be the compact second countable abelian group defined by $\Pi_{i \in \mathbf{N}}\{0,1,2\}_{i}$ where the group structure is defined by coordinate-wise addition mod 3 . We note that every element of $A$ except the identity has order 3. By our remarks in Example 4.4 we can embed $A_{3}$ into $H^{1}\left((Y, G) ; S^{1}\right)$. Indeed let $\left(X, \mu, \hat{A}_{3}\right)$ be as in the example, and let $\Phi:(Y, \nu) \rightarrow(X, \mu)$ be an orbit equivalence, so that there exists a map 
$\Lambda:(Y \times G) \rightarrow\left(X \times \hat{A}_{3}\right)$ given by $\Lambda(y, g)=(\Phi(y), \varphi(y, g))$ where $\varphi \in Z^{1}\left((Y, G) ; \hat{A_{3}}\right)$. Then for any $\rho \in \hat{\hat{A}_{3}}=A_{3}, \rho \circ \varphi \in Z^{1}\left((Y, G) ; S_{1}\right)$. This map of $A_{3}$ into $Z^{1}\left((Y, G) ; S^{1}\right)$ is clearly a group homomorphism, and by the remarks of the preceding paragraph it is clear that $\rho \circ \varphi$ is a coboundary if and only if $\rho \equiv 1$.

TheOREM 4.4. Let $(Y, \nu, G)$ and $A_{3}$ be as above. The family $\left\{A_{\rho \circ \varphi}(S(Y, G)) \mid \rho \in A_{3}\right\}$ is an uncountable family of Cartan subalgebras of the hyperfinite $\mathrm{II}_{1}$ factor, no two of which are inner conjugate.

Proof. By Claim 4.1 it is enough to show that $A_{\rho \circ \varphi}(S(Y, G))$ is inner conjugate to $S(Y, G)$ if and only if $\rho \equiv 1$. By Theorem 3.11, $A_{\rho \circ \varphi}(S(Y, G))$ is inner conjugate to $S(Y, G)$ if and only if there exists $\gamma \in \hat{G}$ such that the cocycle $\gamma(y, g)=\gamma(g)$ is cohomologous to $\rho \circ \varphi$. Notice that $\rho \circ \varphi$ has order 3 in $H^{1}\left((Y, G) ; S^{1}\right)$ unless $\rho \equiv 1$ in which case $\rho \circ \varphi$ is obviously trivial. On the other hand, every element of $\hat{G}$ has order two, so that for $\gamma \in \hat{G}$, the cocycle $\gamma(y, g)=\gamma(g)$ has order at most two in $H^{1}\left((Y, G) ; S^{1}\right)$. In fact $\gamma(y, g)$ has order one if $\gamma$ is an eigencharacter for some eigenfunction of $(Y, \nu, G)$ and order two if not. Recalling that $\rho \circ \varphi$ will be cohomologous to $\gamma(y, g)$ if and only if $[\rho \circ \varphi]=[\gamma]$ in $H^{1}\left((Y, G) ; S^{1}\right)$, this order argument implies that $\rho \circ \varphi$ is cohomologous to some $\gamma(y, g)$ if and only if $\rho \circ \varphi$ is a coboundary (hence $\gamma$ is an eigencharacter for $(Y, \nu, G))$. In this case $\rho \equiv 1$. Therefore $A_{\rho \circ \varphi}(S(Y, G))$ is inner conjugate to $S(Y, G)$ if and only if $\rho \equiv 1$ which implies by the claim that the family

$$
\left\{A_{\rho \circ \varphi}(S(Y, G)) \mid \rho \in A\right\}
$$

is a family of pairwise non-inner-conjugate Cartan subalgebras of the hyperfinite $\mathrm{II}_{1}$ factor. Since $A$ has the cardinality of the continuum, this completes the proof.

REMARK 4.5. Let the action of $\mathbf{Z}$ on $\left(S^{1}, \nu_{S^{1}}\right)$ be generated by multiplication by $\lambda=e^{2 \pi i \alpha}, \alpha$ irrational. Then $\left(S^{1}, \nu, \mathbf{Z}\right)_{\lambda}$ has pure point spectrum. In [17] it was claimed that there exist an uncountable family of cocycles of $1 \subset Z^{1}\left(\left(S^{1}, \mathbf{Z}\right)_{\lambda} ; S^{1}\right)$ such that for $\beta_{1}, \beta_{2} \in I, A_{\beta_{1}}\left(S\left(S^{1}, Z\right)_{\lambda}\right)$ is inner conjugate to $A_{\beta_{2}}\left(S\left(S^{1}, Z\right)_{\lambda}\right)$ if and only if $\beta_{1}=\beta_{2}$. To obtain $I$ one embeds $A_{2}=\Pi_{i \in \mathbf{N}}\{0,1\}_{i}$ into $H^{1}\left(\left(S^{1}, \mathbf{Z}\right)_{\lambda} ; S^{1}\right)$ as in Example 4.4. Then one shows there exists a subgroup $L \subset K_{2}$ with $\left[K_{2}: L\right] \leq 4$ such that $\left\{A_{\beta}\left(S\left(S^{1}, \mathbf{Z}\right)_{\lambda}\right) \|[\beta] \in L\right\}$ is the desired family. This proof is somewhat more complicated than the construction given in Theorem 4.4; one must pass to the subgroup $L$ due to the fact that there are three distinct abelian skew product ergodic extensions of $\left(S^{1}, \nu, \mathbf{Z}\right)_{\alpha}$ by $D_{2}=\{1,-1\}$ 
having pure point spectrum, which correspond to the three distinct subgroups of $S^{1}$ containing $\left\{\lambda^{n} \mid n \in \mathbf{Z}\right\}$ as a subgroup of index 2. These extensions must be taken into account when applying Theorem 3.8.

Note added in proof. After the publication of [17] and after writing this article, the author received a preprint from S. Kawakami, "On the weak cohomology groups..." to appear in Proc. 3rd Japan U. S. Seminar, which contained results similar to some of the results in our $\S \S 3$ and 4, and an article by V. Jones and S. Popa, Some properties of M.A.S.A.s in factors, Birk. Verlag Basel, 0T6 (1982), 89-102, in which results related to the topic of our $\S 4$ are proved. Also see another article of G. Muravera, On regular abelian subalgebras, Sib. Math. J. 13 (1982), 805-816, in which an uncountable family of elements in the hyperfinite $\mathrm{II}_{1}$, factor is constructed, no two of which are inner conjugate, and each of which generates a regular M.A.S.A.

\section{REFERENCES}

[1] A. Connes, J. Feldman, and B. Weiss, Amenable equivalence relations are generated by a single transformation, preprint (1980).

[2] A. Connes and V. Jones, $A \mathrm{II}_{1}$ factor with two non-conjugate Cartan subalgebras, Bull. Amer. Math Soc. (New Series) 6 (1982), 211-212.

[3] J. Dixmier, Sous-anneaux abeliens maximaux dans les facteurs de type fini, Ann. of Math., 59 (1954), 279-286.

[4] H. A. Dye, On groups of measure-preserving transformations, I, Amer. J. Math., 81 (1959), 119-159, II, Amer. J. Math., 85 (1963), 551-576.

[5] J. Feldman and C. C. Moore, Ergodic equivalence relations, cohomology, and von Neumann algebras I, Trans. Amer. Math. Soc., 234 (no. 2) (1977), 289-324.

[6] Ergodic equivalence relations, cohomology, and von Neumann algebras II, Trans. Amer. Math. Soc., 234 (no. 2) (1977), 325-359.

[7] A. Guichardet, Une charactérisation des algebras de von Neumann discretes, Bull. Soc. Math France, 89 (1961), 77-101.

[8] P. Hahn, Reconstruction of factors from measures on Takesaki's equivalence relation, J. Functional Anal., 31 (1971), 263-271.

[9] A. Kirillov, Dynamical systems, factors, and representations of groups, Usphehi Math. Nauk., 22 (1967), 63-75.

[10] G. W. Mackey, Point realization of transformation groups, Illinois J. Math., 6 (1962), 327-335.

[11] _ Ergodic theory and virtual groups, Math. Annalen, 166 (1966), 187-207.

[12] Ergodic theory and its significance for statistical mechanics and probability theory, Advances in Math., 13 (1974), 176-269.

[13] G. P. Muravera, Maximal abelian subrings in an approximately finite factor, Siberian Math. J., 9 (1968), 614-622.

[14] M. Nakamura and Z. Takeda, On some elementary properties of the crossed product of von Neumann algebras, Proc. Japan Acad., 34 (1958), 489-494.

[15] _ On inner automorphisms of certain finite factors, Proc. Japan Acad., 37 (1961), 31-33. 
[16] O. A. Nielsen, Maximal abelian subalgebras of hyperfinite factors II, J. Funct. Anal., 6 (1970), 192-202.

[17] J. Packer, On the normalizer of certain subalgebras of group measure factors, Bull Amer. Math. Soc. (New Series) 7, 397-402.

[18] A. Ramsey, Virtual groups and group actions, Advances in Math., 6 (1971), 253-322.

[19] J. Renault, $A$ Groupoid Approach to $C^{*}$-Algebras, Lecture Notes in Math, vol. 793, Springer-Verlag, Berlin and New York, 1980.

[20] C. Series, Ergodic actions of product groups, Ph. D. Thesis, Harvard University, 1976.

[21] I. Singer, Automorphisms of finite factors, Amer. J. Math., 77 (1955), 117-133.

[22] M. Takesaki, On the unitary equivalence among components of decomposition of representations of involutive Banach algebras and the associated diagonal algebras, Tôhoku Math. J., 25 (1963), 365-393.

[23] M. Takesaki, Theory of Operator Algebras I, Springer-Verlag, Berlin and New York, 1979.

[24] J. Westman, Virtual group homomorphisms with dense range, Illinois J. Math., 20 (1976), 41-47.

[25] G. Zeller-Meier, Products croisés d'une $C^{*}$-algebre par un group d'automorphismes, J. Math Pures Appl., 47 (1968), 101-239.

[26] R. Zimmer, Extensions of ergodic actions, Illinois J. Math., 2 (1976), 373-409.

[27] H. Anzai, Ergodic skew product transformations on the torus, Osaka Math. J., 3 (1951), 83-89.

Received April 4, 1983. The work in this paper forms a portion of the author's doctoral dissertation written at Harvard University under the direction of Professor Georege Mackey. The author is heavily indebted to Professor Mackey for his encouragement and for many useful suggestions and ideas. Some of the results in this paper were announced in [17].

NATIONAL UNIVERSITY OF SingaPORE

KENT RIDGE, Singapore 0511 



\section{PACIFIC JOURNAL OF MATHEMATICS EDITORS}

\author{
V. S. VARADARAJAN (Managing Editor) \\ University of California \\ Los Angeles, CA 90024 \\ Charles R. DePrima \\ California Institute of Technology \\ Pasadena, CA 91125 \\ R. FInN \\ Stanford University \\ Stanford, CA 94305
}

\section{HeRmanN FlaschKa \\ University of Arizona \\ Tucson, AZ 85721}

Ramesh A. Gangolli

University of Washington

Seattle, WA 98195

ROBION KIRBY

University of California

Berkeley, CA 94720

\section{C. MOORE}

University of California

Berkeley, CA 94720

\section{H. SAMELSON}

Stanford University

Stanford, CA 94305

HAROLD STARK

University of California, San Diego

La Jolla, CA 92093

\section{ASSOCIATE EDITORS}
R. ARENS
E. F. BECKENBACH
B. H. NeumanN
F. Wolf
K. Yoshida (1906-1982)

\section{SUPPORTING INSTITUTIONS}
CALIFORNIA INSTITUTE OF TECHNOLOGY

\author{
UNIVERSITY OF SOUTHERN CALIFORNIA \\ STANFORD UNIVERSITY \\ UNIVERSITY OF HAWAII \\ UNIVERSITY OF TOKYO \\ UNIVERSITY OF UTAH \\ WASHINGTON STATE UNIVERSITY \\ UNIVERSITY OF WASHINGTON
}
UNIVERSITY OF OREGON

\begin{abstract}
UNIVERSITY OF ARIZONA
UNIVERSITY OF BRITISH COLUMBIA

UNIVERSITY OF CALIFORNIA

MONTANA STATE UNIVERSITY

UNIVERSITY OF NEVADA, RENO

NEW MEXICO STATE UNIVERSITY

OREGON STATE UNIVERSITY
\end{abstract}

The Supporting Institutions listed above contribute to the cost of publication of this Journal, but they are not owners or publishers and have no responsibility for its content or policies.

Mathematical papers intended for publication in the Pacific Journal of Mathematics should be in typed form or offset-reproduced (not dittoed), double spaced with large margins. Please do not use built up fractions in the text of the manuscript. However, you may use them in the displayed equations. Underline Greek letters in red, German in green, and script in blue. The first paragraph must be capable of being used separately as a synopsis of the entire paper. In particular it should contain no bibliographic references. Please propose a heading for the odd numbered pages of less than 35 characters. Manuscripts, in triplicate, may be sent to any one of the editors. Please classify according to the scheme of Math. Reviews, Index to Vol. 39. Supply name and address of author to whom proofs should be sent. All other communications should be addressed to the managing editor, or Elaine Barth, University of California, Los Angeles, California 90024.

There are page-charges associated with articles appearing in the Pacific Journal of Mathematics. These charges are expected to be paid by the author's University, Government Agency or Company. If the author or authors do not have access to such Institutional support these charges are waived. Single authors will receive 50 free reprints; joint authors will receive a total of 100 free reprints. Additional copies may be obtained at cost in multiples of 50 .

The Pacific Journal of Mathematics is issued monthly as of January 1966. Regular subscription rate: $\$ 190.00$ a year (5 Vols., 10 issues). Special rate: $\$ 66.00$ a year to individual members of supporting institutions.

Subscriptions, orders for numbers issued in the last three calendar years, and changes of address should be sent to Pacific Journal of Mathematics, P.O. Box 969, Carmel Valley, CA 93924, U.S.A. Old back numbers obtainable from Kraus Periodicals Co., Route 100, Millwood, NY 10546.

The Pacific Journal of Mathematics at P.O. Box 969, Carmel Valley, CA 93924 (ISSN 0030-8730) publishes 5 volumes per year. Application to mail at Second-class postage rates is pending at Carmel Valley, California, and additional mailing offices. Postmaster: Send address changes to Pacific Journal of Mathematics, P.O. Box 969, Carmel Valley, CA 93924.

PUBLISHED BY PACIFIC JOURNAL OF MATHEMATICS, A NON-PROFIT CORPORATION

Copyright $\odot 1985$ by Pacific Journal of Mathematics 


\section{Pacific Journal of Mathematics}

Vol. 119, No. $2 \quad$ June, 1985

Mustafa Agah Akcoglu and Meira Falkowitz (Soshniak), A general local

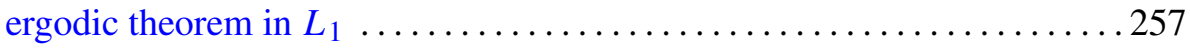

W. Wistar (William) Comfort and Lewis Chandlee Robertson,

Cardinality constraints for pseudocompact and for totally dense

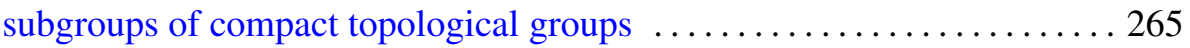

John Morse Delaurentis and Boris G. Pittel, Random permutations and

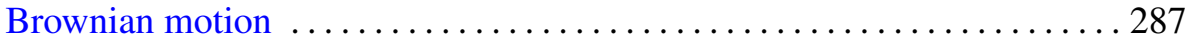

José Esteban Galé, Gel'fand theory in algebras of differentiable functions on Banach spaces ......................................... 303

Harry Gingold, On the location of zeroes of oscillatory solutions of

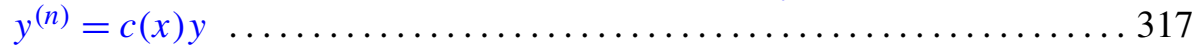

Kei Ji Izuchi, Zero sets of interpolating Blaschke products .............337

Mahesh Nerurkar, Ergodic continuous skew product actions of amenable

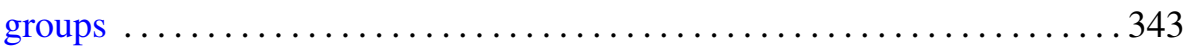

R. Owens, A maximal function characterization of a class of Hardy

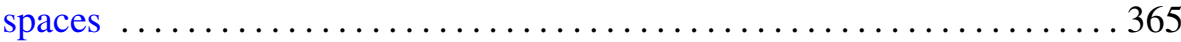

Judith Anne Packer, Point spectrum of ergodic abelian group actions and the corresponding group-measure factors $\ldots \ldots \ldots \ldots \ldots \ldots \ldots \ldots \ldots \ldots \ldots \ldots$

Judith Anne Packer, On the embedding of subalgebras corresponding to quotient actions in group-measure factors $\ldots \ldots \ldots \ldots \ldots \ldots \ldots \ldots . \ldots 4$

Iain Raeburn and Joseph L. Taylor, The bigger Brauer group and étale cohomology

David Rosen, The Diophantine equation $a x+b y=c$ in $Q(\sqrt{5})$ and other number fields

Mau-Hsiang Shih and Kok Keong Tan, Noncompact sets with convex sections

Lee Barlow Whitt, Codimension two isometric immersions between Euclidean spaces

Rodney Ian Yager, Iwasawa theory for the anticyclotomic extension 